\title{
In-Fiber Subpicosecond Pulse Shaping for Nonlinear Optical Telecommunication Data Processing at 640 Gbit/s
}

\author{
J. Azaña, ${ }^{1}$ L. K. Oxenløwe, ${ }^{2}$ E. Palushani, ${ }^{2}$ R. Slavík, ${ }^{3,4}$ M. Galili, ${ }^{2}$ H. C. H. Mulvad, ${ }^{2}$ H. Hu, ${ }^{2}$ \\ Y. Park, ${ }^{1,5}$ A. T. Clausen, ${ }^{2}$ and P. Jeppesen ${ }^{2}$ \\ ${ }^{1}$ Centre Energie, Matériaux et Télécommunications, Institut National de la Recherche Scientifique (INRS), \\ Montreal, QC, Canada H5A 1 K6 \\ ${ }^{2}$ DTU Fotonik, Technical University of Denmark, Ørsteds Square 343, 2800 Lyngby, Denmark \\ ${ }^{3}$ ORC, University of Southampton, Southampton SO17 1BJ, UK \\ ${ }^{4}$ Institute of Photonic Engineering, Academy of Sciences of the Czech Republic, 18251 Prague, Czech Republic \\ ${ }^{5}$ Automated Precision Inc., 15000 Johns Hopkins Drire, Rockville, MD 20850, USA
}

Correspondence should be addressed to L. K. Oxenløwe, lkox@fotonik.dtu.dk

Received 16 June 2011; Accepted 26 September 2011

Academic Editor: Miguel Gonzalez Herraez

Copyright (C) 2012 J. Azaña et al. This is an open access article distributed under the Creative Commons Attribution License, which permits unrestricted use, distribution, and reproduction in any medium, provided the original work is properly cited.

\begin{abstract}
We review recent work on all-fiber (long-period fiber grating) devices for optical pulse shaping, particularly flat-top pulse generation, down to the subpicosecond range and their application for nonlinear switching (demultiplexing) of optical timedivision multiplexed (OTDM) data signals in fiber-optic telecommunication links operating up to 640 Gbit/s. Experiments are presented demonstrating error-free 640-to-10 Gbit/s demultiplexing of the 64 tributary channels using the generated flat-top pulses for temporal gating in a Kerr-effect-based nonlinear optical loop mirror. The use of flat-top pulses has critical benefits in the demultiplexing process, including a significantly increased timing-jitter tolerance (up to $\sim 500 \mathrm{fs}$, i.e., $30 \%$ of the bit period) and the associated improvement in the bit-error-rate performance (e.g., with a sensitivity increase of up to $\sim 13 \mathrm{~dB}$ as compared with the use of Gaussian-like gating pulses). Long-period fiber grating pulse shapers with reduced polarization dependence are fabricated and successfully used for polarization-independent 640 -to-10 Gbit/s demultiplexing experiments.
\end{abstract}

\section{Introduction}

Techniques for the precise synthesis and control of the temporal shape of optical pulses with durations in the picosecond and subpicosecond regimes [1] are of fundamental importance for a wide range of applications in ultrahigh-bitrate optical communications, particularly to enhance the performance of a range of nonlinear optics-based data-processing operations [1-10]. As a very relevant example, (sub)picosecond flat-top (rectangular-like) optical pulses are highly desired in applications requiring the use of a welldefined temporal gating window, for example, for nonlinear time-domain switching of optical telecommunication data. The use of flat-top optical pulses as control/gating pulses in nonlinear time-domain optical switches translates into important advantages as compared with the (more conventional) use of Gaussian-like optical control pulses. Figure 1 illustrates the concept of nonlinear optical switching for temporal demultiplexing of serial optical time-division multiplexed (OTDM) data. For optimum performance, the gating pulse has to be shorter than the one-bit time window and at the same time it should have constant intensity over a time interval as long as possible. Both of these requirements can be fulfilled when using flat-top pulses. Indeed, as illustrated in Figure 1, flat-top optical control pulses increase the tolerance to timing jitter in the system, thus improving the overall performance of the nonlinear switching scheme, for example, leading to a significantly improved receiver sensitivity (needed input signal power to achieve a bit error rate, BER $<$ $\left.10^{-9}\right)[2-6]$.

Flat-top optical pulse waveforms can be generated from Gaussian-like optical pulses (easily generated from modelocked laser systems) using many different pulse-shaping techniques $[1-7,11-15]$. From a practical viewpoint, the 


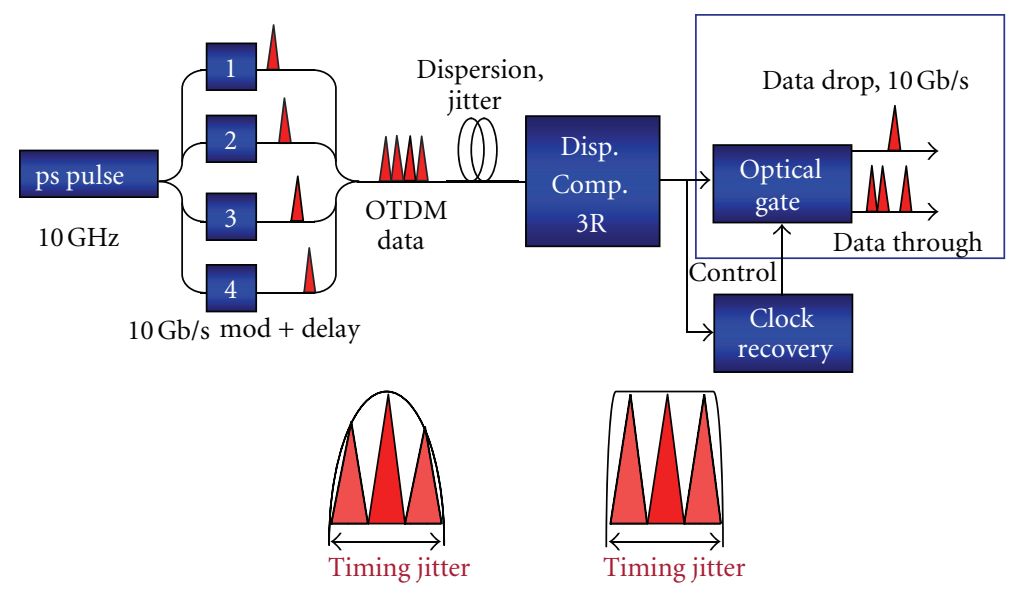

FIGURE 1: General schematic of demultiplexing in OTDM by nonlinear switching and illustration of the timing-jitter tolerance improvement achieved by use of flat-top pulse gating.

two following features are highly desired: (i) linear pulse reshaping techniques are attractive because they generally require a simpler implementation and are independent of the input optical pulse power; (ii) an all-fiber implementation allows for low insertion loss, robust, and low-cost realization with full compatibility with fiber-based systems. Notice that in a linear re-shaping method, the steepness of the rise/decay edges in the synthesized flat-top waveform is limited by the input optical bandwidth. Concerning linear all-fiber flattop temporal pulse shapers, a very interesting method is based on the use of customized in-fiber Bragg (short-period) gratings (FBGs) operating in reflection $[2-4,12]$. A FBG device is essentially a (quasi)periodic perturbation of the fiber refractive index that is permanently photo-induced along the length of a relatively short section of the optical fiber [16]. FBGs are designed to induce coupling between two counter-propagating modes, namely, the same fundamental mode in the fiber as it propagates in two opposite directions, around a target resonance wavelength. Roughly speaking, FBGs behave as wavelength-selective mirrors. For flat-top pulse re-shaping, an FBG is used as a linear filter operating over an input Gaussian-like (or soliton-like) optical pulse and the grating coupling-strength and period profiles are designed to achieve the desired sinc-like spectral transfer function. This pulse-shaping strategy is thus based on the proper manipulation of the spectral-domain features of the input optical pulse in order to obtain the spectral profile that corresponds to the desired temporal profile. This so-called Fourier-domain approach has been extensively used in conventional optical pulse shapers based on nonfiber solutions, namely, bulk diffraction gratings [1] and integrated arrayedwaveguide gratings [11]. The main limitations of FBG-based optical pulse shapers are that (i) a complex amplitude and phase grating profile is usually required and (ii) due to the FBG limited bandwidth, temporal waveforms shorter than a few tens of picoseconds cannot be easily synthesized. This latter limitation is related to the spatial resolution of the grating-strength variation that can be practically photoinscribed along the fiber.
It has been previously anticipated [17] that the two mentioned limitations could be overcome using long-period fiber gratings, LPGs, instead of FBGs. LPGs are based on coupling between two copropagating modes, typically the fiber fundamental (core) mode and any of the fiber's cladding modes [16]. In particular, two different LPG-based concepts have been proposed for optical flat-top pulse shaping. The first one [17] is a general method, eventually enabling the synthesis of almost any desired pulse shape by properly tailoring the grating coupling-strength and period variations. However, this method is difficult to implement in practice since the pulse re-shaping operation typically needs to be realized via a "core mode to cladding mode" coupling or vice versa [18] (i.e., the input and output signals must be in different fiber propagation modes). The second method is based on the use of an ultrafast optical differentiator, that is, a linear optical filter designed to calculate the first time derivative of an incoming arbitrary optical waveform. In particular, it has been shown that flat-top pulse waveforms can be synthesized by propagation of a Gaussian-like optical pulse through an optical differentiator by properly detuning the input pulse central wavelength with respect to the resonance (zerotransmission) wavelength of the differentiator [14]. Ultrafast optical differentiators capable of operation over $\mathrm{THz}$ bandwidths (corresponding to input time features well in the subpicosecond range) can be created using either a single uniform LPG operating in full coupling condition $[19,20]$ or a Mach-Zehnder-(MZ-) type all-fiber interferometer created by concatenation of two ultrabroadband uniform LPGs [21].

LPG-based optical pulse shapers $[14,15,22]$ have enabled the synthesis of flat-top optical pulses well in the subpicosecond range, that is, with full-width-at-half-maximum, FWHM, time widths as short as $\sim 700 \mathrm{fs}$ [23], about 10-20-times shorter than with any other reported all-fiber method, for example, FBG-based pulse shapers. This improvement has in turn enabled the development of a range of nonlinear optical telecommunication data processing schemes enhanced by flat-top pulse shapers at unprecedented bit rates, namely, up to $640 \mathrm{Gbit} / \mathrm{s}$ [24-27]. Error-free 
demultiplexing of OTDM signals with significantly improved timing-jitter tolerances has been demonstrated using LPGbased pulse shapers. This represents a critical advance in the field because for high-speed serial data transmission that operates at rates of $160 \mathrm{Gbit} / \mathrm{s}$ and above, management of the timing jitter becomes extremely important while being increasingly challenging [28-33]. In addition, the LPG pulse shapers can be fabricated to exhibit very low polarization sensitivity $[23,26,27]$; this latter feature can be exploited to create polarization-independent OTDM demultiplexing systems [27].

This work reviews recent progress on the design and fabrication of all-fiber (LPG-based) picosecond and subpicosecond flat-top optical pulse shapers $[14,15,17-22]$ and their application to nonlinear optical telecommunication data processing [23-27], particularly demultiplexing of OTDM serial data using nonlinear optical loop mirror switches with flat-top gating pulses, at bit rates up to $640 \mathrm{Gbit} / \mathrm{s}$. The reviewed set of experiments demonstrate error-free 640-to$10 \mathrm{Gbit} / \mathrm{s}$ data signal demultiplexing over the 64 OTDM channels with a significantly improved performance over the use of Gaussian-like control/gating pulses, for example, in terms of receiver sensitivity (up to $\sim 13 \mathrm{~dB}$, with a penalty over the $10 \mathrm{Gbit} / \mathrm{s}$ back-to-back measurements as low as $\sim 0.5 \mathrm{~dB}$ ), time jitter tolerance (up to $\sim 500 \mathrm{fs}$, corresponding to $\sim 30 \%$ of the OTDM signal bit period), and optimized polarization insensitivity. The paper is structured as follows: Section 2 reviews the principle of the flat-top pulse shaping based on ultrafast optical differentiation Section 2.1 together with two different LPG-based implementations, namely, a single-LPG filter Section 2.2 and a double-LPG MZ-type interferometer Section 2.3. The application of these two pulse-shaping technologies for demultiplexing of 640-Gbit/s OTDM data signals is revisited in Section 3, including the most relevant experiments based on the use of single-LPG pulse shapers Section 3.1 and those based on the use of double-LPG pulse shapers Section 3.2, such as polarizationindependent demultiplexing. Finally, Section 4 outlines the main conclusions and future prospects of the work reviewed here.

\section{Flat-Top Pulse-Shaping Principle and Long-Period Fiber Grating (LPG) Implementations}

\footnotetext{
2.1. Flat-Top Pulse Shaping Based on Optical Differentiation. The flat-top pulse reshaping technique used in the reviewed work is based on a filtering scheme that employs an optical temporal differentiator [14]. It has been anticipated that any desired ultrafast temporal waveform could be synthesized as a linear superposition of a Gaussian-like pulse and its successive time derivatives [34]. The used flat-top pulse reshaping method is based on this general property and in particular, it exploits the fact that a flat-top intensity waveform can be well approximated by only two terms of the general series, that is, by a proper combination of the input Gaussian-like pulse and its first time derivative. In what follows we describe an extremely simple mechanism to implement this idea; this
}

mechanism is based on the use of an optical differentiator where the relative weight between the two required temporal terms is easily adjusted through frequency detuning between the input pulse and the differentiator resonance [14].

Optical temporal differentiation $[19,20]$ (that operates over the complex time-domain envelope of an optical signal) can be realized using a linear filter that has a spectral transfer function proportional to $i\left(\omega-\omega_{0}\right)$, where $\omega_{0}$ is the central frequency of the optical differentiator (frequency of zero transmission), $\omega$ is the angular optical frequency variable, and $i$ is the imaginary unit. When considering an input optical pulse of temporal envelope $u(t)$ (spectrally centred at $\left.\omega_{\text {car }}\right)$, with a resulting output optical pulse of temporal envelope $v(t)$, the differentiator was shown to provide the following filtering function [14]

$$
v(t) \propto \frac{\partial u(t)}{\partial t}+i \Delta \omega u(t),
$$

where $\Delta \omega=\omega_{\mathrm{car}}-\omega_{0}$. Assuming that the input optical pulse is transform limited with a real temporal envelope $u(t)$, the temporal intensity profile of the signal at the optical differentiator output can be written as follow:

$$
|v(t)|^{2} \propto\left|\frac{\partial u(t)}{\partial t}\right|^{2}+\Delta \omega^{2}|u(t)|^{2} .
$$

The whole principle, described in what follows, is schematically shown in Figure 2. For $\omega_{\text {car }}=\omega_{0}(\Delta \omega=0)$ the device operates as an optical differentiator. However, for $\omega_{\text {car }} \neq \omega_{0}$, the output $|v(t)|^{2}$ consists of a sum of the differentiated waveform and the original waveform, with a relative weight given by the detuning factor $\Delta \omega^{2}$. When $u(t)$ is a temporally symmetric pulse (e.g., Gaussian-like waveform), the differentiated pulse is a symmetric double-pulse [15]. Hence, the resulting output intensity waveform is a superposition of the differentiated waveform (double-pulse intensity profile, first term in (2)) and the original Gaussian pulse intensity profile (second term in (2)), where the Gaussian pulse intensity peak coincides with the energy valley in the double-pulse waveform. The amount of energy of the original Gaussian pulse that is present in the generated pulse is proportional to the frequency detuning factor $\Delta \omega$. As the frequency detuning $\Delta \omega$ increases, the differentiated pulse is gradually reshaped in such a way that the central valley of the temporal double-pulse intensity is filled by the residual original Gaussian profile, leading to the formation of a single flat-top pulse for an optimal frequency detuning. Generally, the generated flat-top optical pulse is not transform-limited, that is, the pulse temporal profile exhibits a certain nonlinear phase variation. This poses no problem with applications in which only the intensity profile is of interest, for example, nonlinear optical switching experiments based on the Kerreffect (e.g., cross-phase modulation, XPM) in which the flattop pulse waveform is used as the pump pulse.

Unlike other fiber pulse-shaping techniques, for example, based on Fourier filtering [1, 11-13], the optical differentiation-based scheme does not require precise control of the relation between the input pulse and the filter characteristics in terms of bandwidth, provided the differentiator bandwidth is larger than that of the input pulse. This gives an 


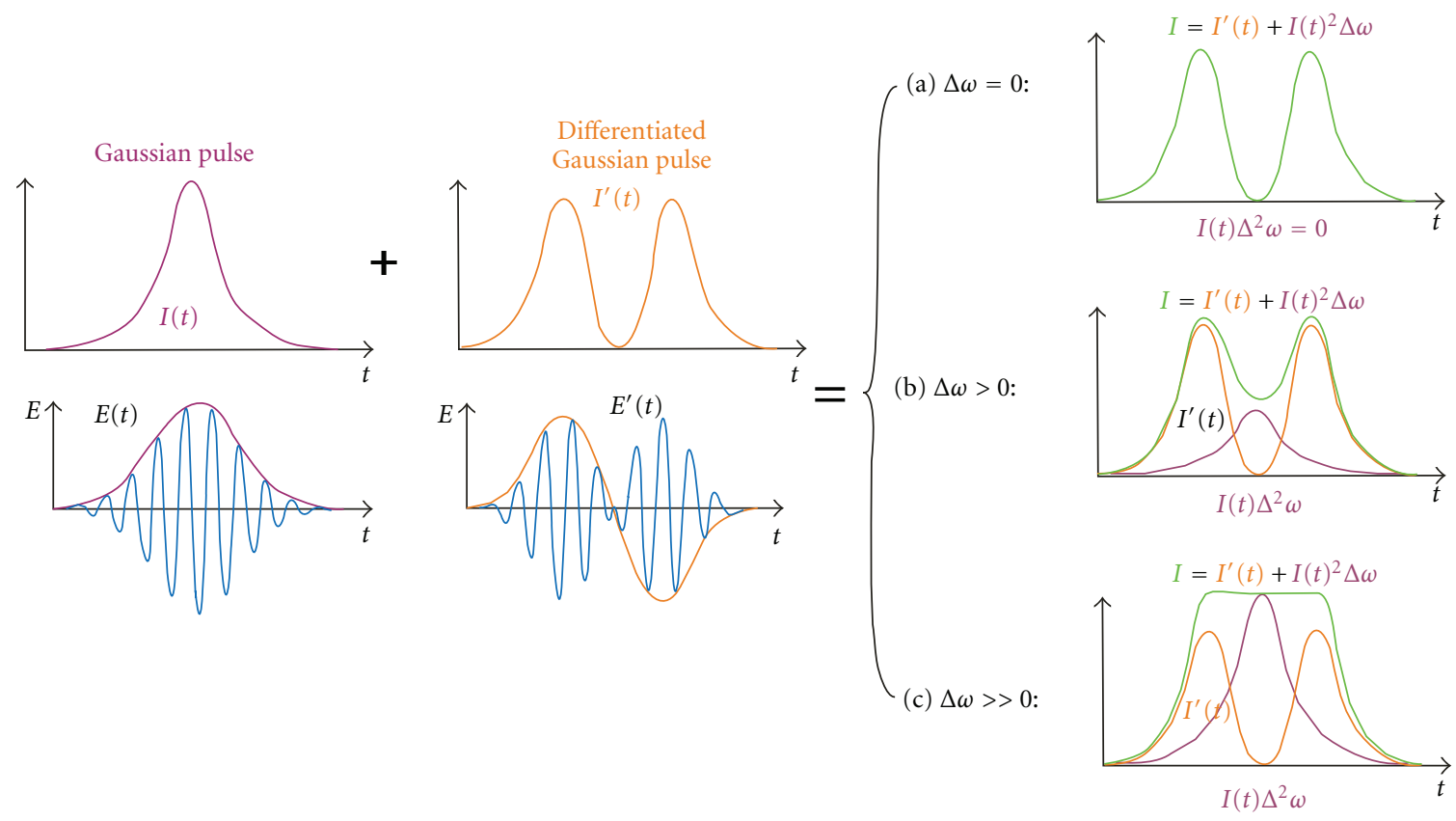

FIGURE 2: Principle of flat-top pulse generation using a frequency-detuned optical differentiator, $\Delta \omega=\omega_{\text {car }}-\omega_{0}$.

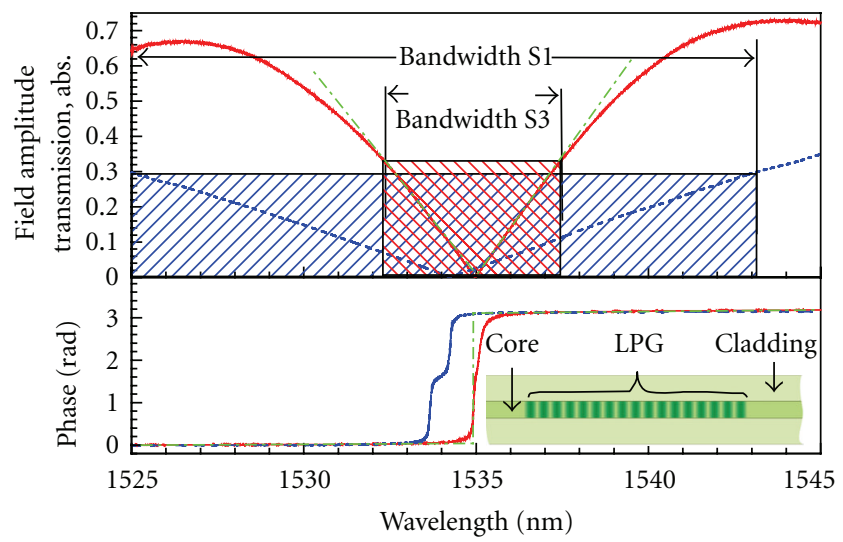

FIGURE 3: Amplitude and phase characteristics of the fiber LPG filters. Measured field amplitude and phase characteristics of the realized long, S3 (red) and short S1 (blue) LPGs together with the theoretical characteristics of an ideal differentiator similar to S3 (green, dash-dotted lines). The S3 and S1 LPG operational bandwidths (highlighted in the figure) are $5.5 \mathrm{~nm}$ and $19 \mathrm{~nm}$, respectively. The inset shows a fiber uniform LPG, where the level of green corresponds to the refractive index.

additional flexibility as a single differentiation device can be used with different short-pulse sources emitting pulses of various durations and shapes. The rise/fall edges of the flat-top pulse are determined by the rise/fall edges of the input pulse (more specifically, by its differential), while the duration of the flat-top pulse is scaled with the duration of the input pulse. As a result, flat-top pulses of different durations can be generated from the same differentiation device by simply tuning the time width of the input optical pulse (this also requires a proper adjustment of the pulseLPG frequency detuning) [14].
2.2. Implementation Based on a Single-LPG Optical Differentiator. In a first implementation, we created the optical differentiator using a single uniform LPG $[19,20]$. As discussed above, an optical temporal differentiator is essentially a linear filtering device providing a spectral transfer function proportional to $i\left(\omega-\omega_{0}\right)$. Consequently, the two key features of the filter's transmission are (i) it depends linearly on the base-band $z$ frequency, and (ii) it is zero at the signal central frequency $\omega_{0}$. These two key features imply an exact $\pi$ phase shift across the central frequency $\omega_{0}$. The ideal complex transmission of an optical differentiator is schematically shown in Figure 3.

The required energy depletion at the signal central frequency can be produced by resonance-induced complete energy transfer elsewhere. Specifically, in fiber optics, this can be achieved by resonant transfer of light between two modes of the optical fiber waveguide. Resonant light coupling is induced when the light propagates through the two interacting modes with identical speeds, which is practically attainable, for example, by an increase or decrease of the light speed in one of the modes using a suitable phase diffraction grating [16]. The specific diffraction grating used in our experiments, which is realized as a periodic change of the refractive index along the direction of light propagation within a single optical fiber, induces resonant coupling between two co-propagating modes and is commonly called long-period fiber grating (LPG) [35]. The term "long" refers to its period, which typically varies from tens to hundreds of micrometers, as opposed to short-period gratings (fiber Bragg gratings, FBGs), where the light is backscattered, resulting in coupling between modes traveling in opposite directions (in a Bragg geometry, the corrugation period is approximately half the optical wavelength, micrometers, or less). 
The phase matching condition in order to achieve mode coupling between the core mode and the forward propagating cladding modes is given by

$$
\beta_{01}-\beta_{\mathrm{cl}}^{(n)}=\frac{2 \pi}{\Lambda},
$$

where $\beta_{01}$ and $\beta_{\mathrm{cl}}^{(n)}$ are, respectively, the propagation constants of the core and the $n$ th-cladding mode, and $\Lambda$ is the periodicity of the grating. The ratio of power coupled into the $n$ th-cladding mode to the initial power contained in the core mode is given by

$$
\frac{P_{\mathrm{cl}}^{(n)}(L)}{P_{01}(0)}=\frac{\sin ^{2}\left[\kappa L \sqrt{1+(\delta / \kappa)^{2}}\right]}{1+(\delta / \kappa)^{2}},
$$

where $\delta$ is the detuning parameter

$$
\delta=\frac{1}{2}\left[\beta_{01}-\beta_{\mathrm{cl}}^{(n)}-\frac{2 \pi}{\Lambda}\right],
$$

$L$ is the grating length, and $\kappa$ is the rate of the induced coupling per unit length between the core guided mode and cladding mode(s) [35]. To obtain efficient coupling between these modes, the period of the LPG must be properly adjusted to cause light diffraction from the core mode into the chosen cladding mode. Due to the different dispersion slopes of these two modes, the resonant coupling occurs only at a specific frequency $\omega_{0}$, referred to as the LPG resonance frequency. It is known that if the device is designed to exactly satisfy the condition $\kappa L=\pi / 2$ then the grating induces a total $(100 \%)$ energy coupling from the input guided core mode into the cladding mode (at $\left.\omega_{0}\right)$. An optical fiber-based LPG specifically designed to provide $100 \%$ coupling between the fiber core mode and one of its cladding modes at the resonance frequency provides both the required $\pi$ phase shift and the transmission linear dependence that is necessary for time differentiation (assuming that the input optical signals are centered at the LPG resonance frequency) [19]. Such an LPG, coincidentally, has the required spectral linear response over a bandwidth as broad as several terahertz [20].

To implement an LPG-based optical differentiator, stringent control of the fiber LPG coupling strength (which must be fixed exactly at $\kappa L=\pi / 2$ ) is required. The transmission spectral responses of two LPG samples fabricated for optical differentiation are reported in Figure 3. These samples were made in a standard fiber sample (SMF-28, Corning Inc.), using the established point-by-point technique with a $\mathrm{CO}_{2}$ laser [36], and they have physical lengths of $2.6 \mathrm{~cm}$ (sample $\mathrm{S} 1)$ and $8.9 \mathrm{~cm}$ (sample S3), respectively, and a grating period of $415 \mu \mathrm{m}$. To perform a very fine tuning of the grating strength (coupling coefficient), we used a technique which takes advantage of the coupling coefficient dependence on the fiber longitudinal strain [37].

The amplitude and phase characteristics of the fiber LPG samples were measured by an Optical Vector Analyzer (Luna Technologies), and are shown in Figure 3. The linear and quadratic terms in the phase curve-caused by the delay and linear chromatic dispersion, respectively-have been

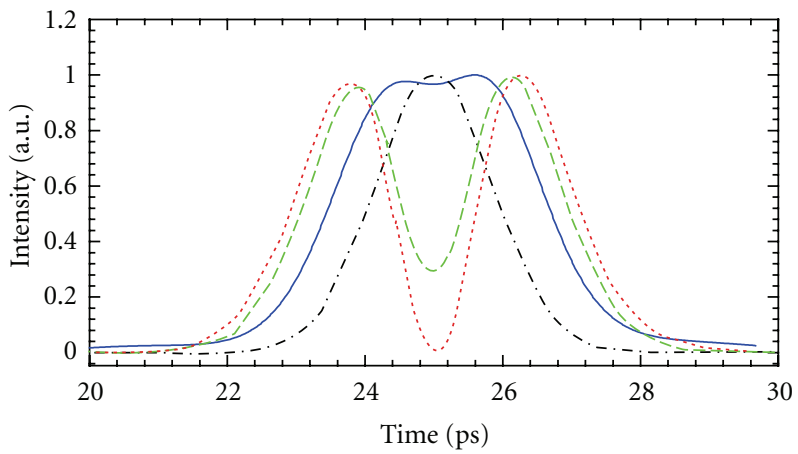

(a)

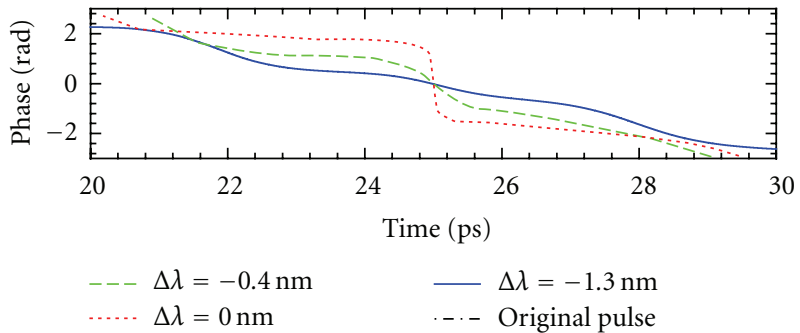

(b)

FIGURE 4: Experimentally generated intensity (a) and phase (b) temporal profiles of the synthesized waveforms at the uniform LPG output when a 1.8 ps input pulse is launched at the input for different input pulse-LPG wavelength detuning $(\Delta \lambda)$ conditions.

subtracted. We measured an exact $\pi$ phase shift at the filter resonance wavelength (1535 $\mathrm{nm}$ for S1, $1534 \mathrm{~nm}$ for S3), which is an essential feature to obtain the desired filtering operation. The LPGs exhibited an extremely deep attenuation, breaking the $60 \mathrm{~dB}$ limit, confirming operation at almost exact full-coupling condition, as required by our application. The usable ("operational") bandwidths of the fabricated LPGs were approximately $19 \mathrm{~nm}$ (S1) and $5.5 \mathrm{~nm}$ (S3). Notice that the "operational" bandwidth is the LPG resonance bandwidth over which the fiber filter provides the desired filtering function (i.e., a linear function of frequency). This corresponds approximately to the bandwidth over which the LPG transmission (in intensity) is lower than $10 \%$.

As discussed in Section 2.1, for flat-top pulse generation, the LPG resonance frequency must be properly detuned from the carrier frequency of the input Gaussian-like pulse [14]. Figure 4 shows experimental results on the formation of a flat-top optical pulse via propagation of a nearly-transformlimited Gaussian-like pulse through the LPG sample S3 reported above with a proper frequency (wavelength) detuning [14]. The input pulses in the experiment shown in Figure 4 were Gaussian-like optical pulses generated from a passively mode-locked wavelength-tunable fiber laser (Pritel Inc.) with full-width-half-maximum (FWHM) pulse width of $1.8 \mathrm{ps}$. The pulses from the laser were first launched into a polarization controller, as the fiber LPG was slightly birefringent [38], and were subsequently propagated through the LPG-based pulse shaper. The output spectrum was monitored using 


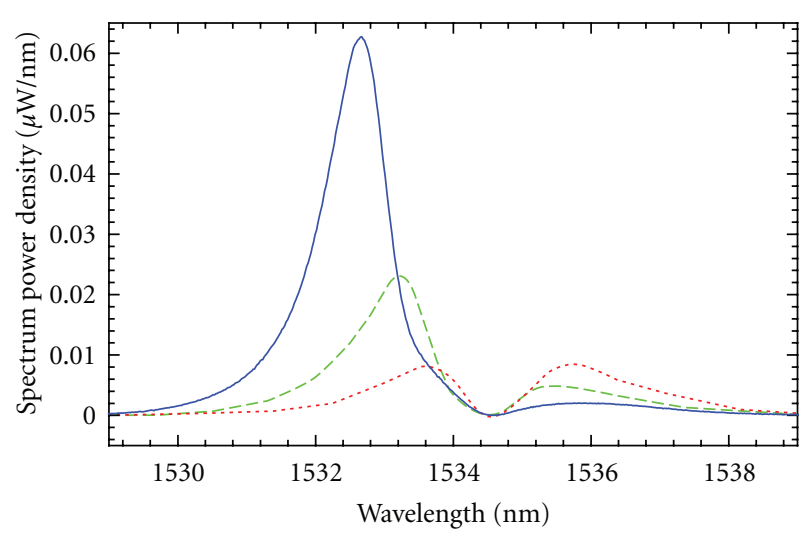

FIGURE 5: Measured spectra corresponding to the synthesized waveforms in Figure 4.

an optical spectrum analyzer (OSA). We used a fiber-based Fourier-transform spectral interferometry (FTSI) setup to retrieve the complex temporal waveform of the output pulse (in this setup, the input pulse was used as the reference pulse) $[39,40]$. FTSI allowed us to monitor the obtained temporal waveform and optimize the experiment conditions, namely, wavelength detuning (in our experiment, this was achieved via fiber laser tuning) and slight adjustment of the LPG coupling coefficient (via LPG straining), so that to achieve the desired flat-top temporal waveform. Figures 4(a) and 4(b) show the amplitude and phase temporal profiles recorded at the LPG output for different values of the pulseLPG wavelength detuning, $\Delta \lambda=0$ (dotted, red curves), $-0.4 \mathrm{~nm}$ (dashed, green curves), and $-1.3 \mathrm{~nm}$ (solid, blue curves), as well as the initial Gaussian pulse (dash-dotted, black curve). The measured spectra corresponding to the three shown temporal waveforms are shown in Figure 5. The estimated FWHM time width of the experimentally generated flat-top waveform (for $\Delta \lambda=-1.3 \mathrm{~nm}$ ) is $\approx 3.2 \mathrm{ps}$. The energetic efficiency of the performed filtering process (ratio of the output power to the input power) was measured to be $5.5 \%$. Energetic efficiency can be increased by use of an LPG with a narrower resonance bandwidth, that is, approaching the input pulse bandwidth [19].

Due to the large bandwidth inherently provided by LPGs, this technique is well suited for pulse re-shaping operations down to the subpicosecond regime and as a result, it is easily scalable to ultrahigh bit rates (e.g., $640 \mathrm{Gbit} / \mathrm{s}$ ). In addition, as mentioned above, flat-top pulses of different durations can be achieved from the same LPG pulse shaper by simply modifying the input pulse time-width and the corresponding pulse-LPG frequency detuning. For instance, 1ps (FWHM) flat-top pulses were generated using this same LPG sample, S3, from $\sim 600$ fs Gaussian-like input pulses [14]. Using numerical simulations, we have estimated that flat-top waveforms with time widths down to $\approx 550 \mathrm{fs}$ (from $\approx 290$-fs input Gaussian-like pulses) could be efficiently synthesized using this LPG sample, S3. Shorter flat-top pulses could be generated using an LPG with a broader resonance bandwidth, for example, sample $\mathrm{S} 1$.

As another interesting advantage of the single LPG flattop pulse re-shaping method, we have shown that a consid- erable group-velocity dispersion-induced distortion on the flat-top pulse profile, for example, as caused by propagation through a few meters of standard SMF, can be compensated through a simple axial strain applied along the LPG [15].

Notice that the above reported LPG was photo-inscribed from one of the sides of the optical fiber. This procedure yields nonuniform refractive index change across the fiber cross-section, which typically introduces a slight photoinduced birefringence [38]. This causes the resonance frequency to vary depending on the polarization of the input light. Fiber illumination simultaneously from three sides of the fiber can be achieved using two-mirrors-assisted $\mathrm{CO}_{2}$ laser radiation [41]. This configuration yields azimuthally symmetric illumination of the fiber, greatly reducing the photo-induced birefringence in the LPG. More discussions on this important practical issue, including detailed evaluation of the LPG resonance birefringence, can be found in Section 3.1 below.

\subsection{Implementation Based on a Double-LPG Optical Differen-} tiator. Optical differentiation can be achieved using a twoarm (e.g., Mach-Zehnder, MZ-type) interferometer operated around any of its destructive interference frequencies [42]. Such an interferometer device can be created in an all-fiber format using two concatenated uniform LPGs [21]. This configuration offers an increased stability against environmental fluctuations and the potential for ultra-broadband operation bandwidths, well in the $\mathrm{THz}$ range. In what follows, we review the principle of operation of a two-arm interferometer as an optical differentiator and its implementation using concatenated LPGs.

A symmetric (splitting/coupling ratio of $50 \%$ ), unbalanced (the two interfering paths are of different lengths) interferometer has the following spectral transfer function:

$$
H_{t}\left(\omega-\omega_{0}\right) \approx 1+\exp (i \omega \tau)=1+\exp \left[i\left(\omega-\omega_{0}\right) \tau\right] \exp \left(i \omega_{0} \tau\right)
$$

where $\tau$ is the relative time delay between the two interferometer arms. Setting the interferometer to operate at a minimum transmission $\left(H_{t}\left(\omega-\omega_{0}\right)=0\right)$ at the carrier frequency $\left(\omega=\omega_{0}\right)$, it follows from (6) that

$$
\tau=\frac{\pi(2 m+1)}{\omega_{0}}
$$

where $m$ is an arbitrary integer. Introducing this value into (6) we obtain:

$$
H_{t}\left(\omega-\omega_{0}\right) \approx 1-\exp \left[\frac{i \pi(2 m+1)\left(\omega-\omega_{0}\right)}{\omega_{0}}\right] .
$$

The function in (8) can be approximated over a sufficiently narrow bandwidth centered at $\omega_{0}$ by the first terms of the Taylor series expansion:

$$
\begin{gathered}
\cos \left(\frac{\pi(2 m+1)\left(\omega-\omega_{0}\right)}{\omega_{0}}\right) \cong 1 \\
\sin \left(\frac{\pi(2 m+1)\left(\omega-\omega_{0}\right)}{\omega_{0}}\right) \cong \frac{\pi(2 m+1)\left(\omega-\omega_{0}\right)}{\omega_{0}},
\end{gathered}
$$




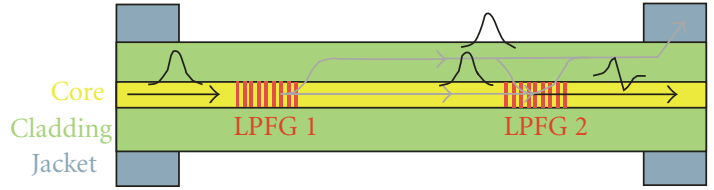

Figure 6: Schematics of the LPG-based common path interferometer for flat-top pulse shaping. The light is split by the first LPG, then propagates in the core and cladding modes with different speeds and is superimposed coherently using the second LPG.

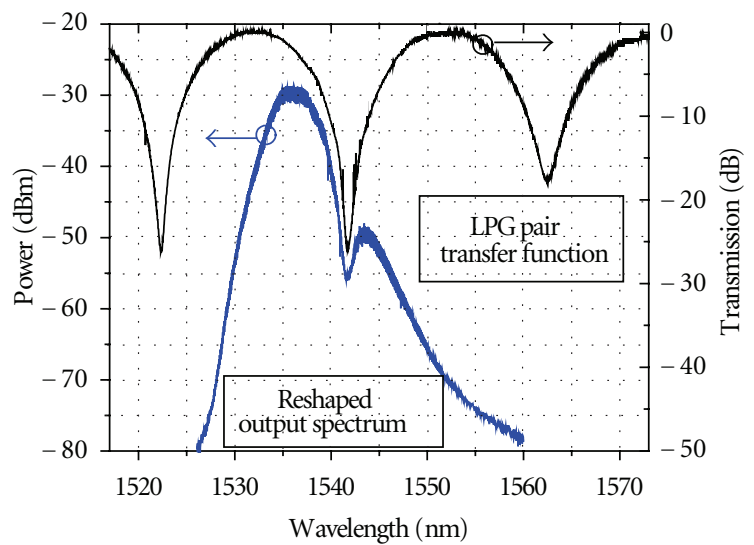

Figure 7: Transfer function of the LPG filters pair and reshaped spectrum of a $700 \mathrm{fs}$ Gaussian-like pulse detuned $<4 \mathrm{~nm}$ away from the destructive interference wavelength of the LPG pair.

resulting in

$$
H_{t}\left(\omega-\omega_{0}\right) \approx \frac{-i\left(\omega-\omega_{0}\right) \pi(2 m+1)}{\omega_{0}}=-i\left(\omega-\omega_{0}\right) \tau,
$$

which is the transmission function required for optical temporal differentiation. As follows from the above discussion, the expression in (10) strictly holds over a narrow bandwidth of frequencies (around $\left.\omega_{0}\right)$ that satisfy the condition $(\omega-$ $\left.\omega_{0}\right) \ll \pi / \tau$. Said another way, a broader differentiation bandwidth can be achieved by decreasing the relative time delay in the interferometer. However, as implied by (10), a reduced relative delay translates into a larger attenuation (higher insertion loss) in the interferometer spectral transfer function, leading to a decreased energetic efficiency in the device operation.

This symmetric unbalanced MZ interferometer device can be created using an all-fiber device consisting of a cascade of two LPGs, Figure 6 [21]. The light is split by the first LPG, then propagates in the core and cladding modes with different speeds and is superimposed coherently using the second LPG. The key in obtaining optical differentiation is a precise symmetry of the MZ interferometer in terms of the splitting ratios in order to obtain full destructive interference. The first LPG couples $50 \%$ of light into a cladding mode. Subsequently, $50 \%$ of energy propagates in the cladding mode while the other $50 \%$ propagates in the core mode. The light propagating in the core mode accumulates (with respect to the cladding-propagating portion of the signal) a delay $\tau$ due to the difference in the group velocities between the core and cladding modes. As both modes propagate within the same fiber and as the interferometer length is typically of the order of a few to tens of centimeters, any environmental change influences both modes in nearly the same manner, which results in a very robust device operation. Moreover, as mentioned above, the LPGs can be fabricated so that their performance is almost independent on the input polarization [41]. In this way, the filter can be made very weakly polarization dependent.

As discussed, for flat-top pulse generation, a nearly transform-limited Gaussian-like optical pulse with a central frequency properly detuned with respect to the differentiator resonance (destructive interference) frequency must be launched at the device input. Figure 7 reports the spectral transfer function of a pair of cascaded LPGs, inscribed in a conventional telecommunication standard SMF, which have a period of $387 \mu \mathrm{m}$ and are spaced $28 \mathrm{~mm}$ apart [27]. This leads to a time delay of $390 \mathrm{fs}$ between the recombined cladding and core pulses. This pair of LPGs was inscribed using two-mirrors-assisted $\mathrm{CO}_{2}$ laser side radiation, considerably reducing the photoinduced birefringence. After inscription of the first LPG with resonant attenuation slightly over $3 \mathrm{~dB}$ (typically $3.2 \mathrm{~dB}$ ) we moved the translation stage by a distance $L$ and inscribed the second LPG. During inscription of the second LPG, the transmission was monitored along both axes of birefringence after each period inscribed (using a superluminescent light-emitting diode, fiber polarizer, polarization controller, and optical spectrum analyzer) and the inscription process was stopped when the resonant attenuation of two consecutive MZ interference destructive interference wavelengths (that appeared around the LPG resonance wavelength) attained its maximum valuetypically more than $30 \mathrm{~dB}$. As the coupling strength of LPGs was slightly strain dependent, the strain applied to the fiber with the two LPGs inscribed was used subsequently to maintain the point of operation.

Figure 8 shows the simulated output pulse intensity profiles for different input pulse-LPG detuning factors, when the input pulse is a $700 \mathrm{fs}$ Gaussian (almost double the inter-modal relative time delay) [27]. Figure 8 displays the fully destructive interference case (trace 1), with a central dip in the pulse shape and spectrum (the device is working as an optical differentiator [5]), and the partially destructive interference case (traces 2-3), where the dip gets filled up to achieve a flat-top pulse. Tuning the input pulse wavelength to an offset $<4 \mathrm{~nm}$ from the destructive interference wavelength of the LPG interferometer leads to formation of a flat-top pulse shape, as seen in trace 3 . The corresponding measured output spectrum is represented in Figure 7. This corresponds to a generated $\sim 1.2$ ps flat-top pulse centered at $1536 \mathrm{~nm}$.

Notice that a similar LPG configuration (cascaded LPG device illustrated in Figure 6) can be used for transformlimited flat-top pulse generation [22]. To achieve a transform-limited pulse at the system output, the input pulse time width must be precisely fixed according to the interferometer relative delay; in addition, the input pulse must be spectrally centered at a constructive-interference frequency of the 


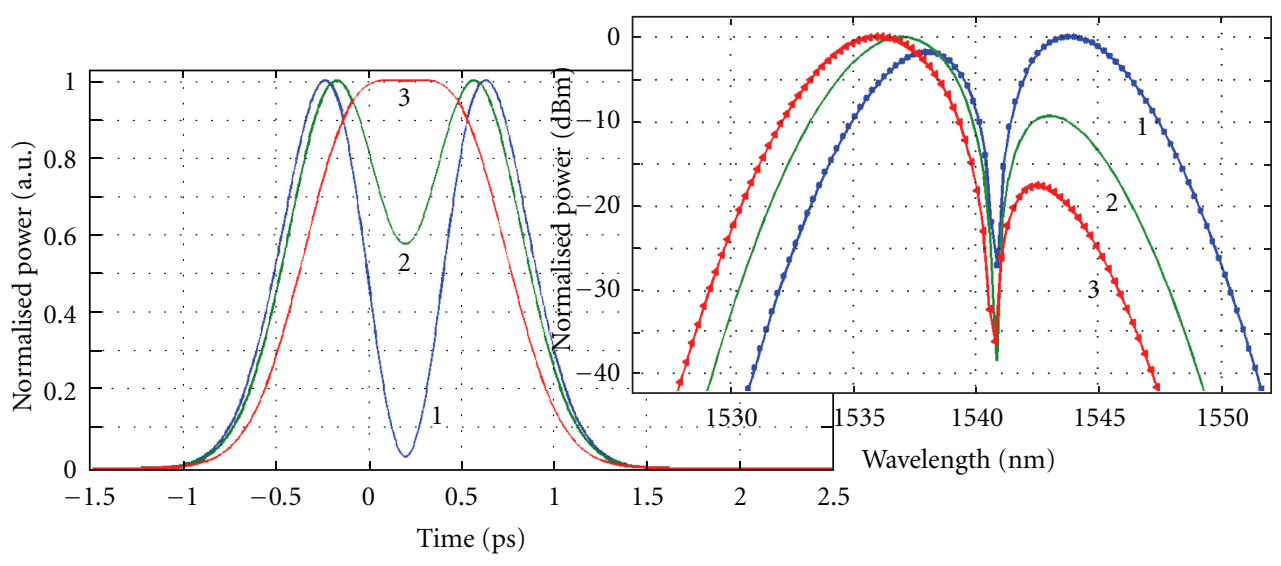

FIGURE 8: Traces in ascending order of pulse intensity profiles (left) and respective spectra (right) for detuning of the signal input wavelength away from the interference fringe of the cascaded LPG filter pair.

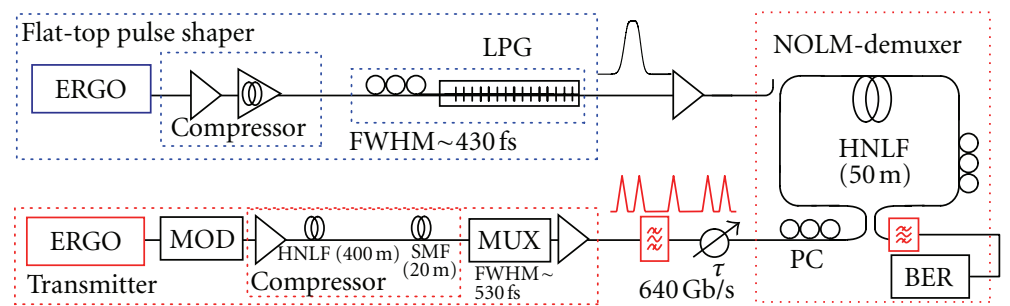

FIGURE 9: Setup for 640 Gbit/s. ERGO—erbium glass oscillator, PC—polarization controller, MOD—modulator, BER—bit error rate.

device. Different pulse waveforms could be generated using this same pulse coherent superposition concept by simply changing the interpulse relative delay [13]. In another recent experiment, a similar double-LPG device was used to generate parabolic-like optical pulses aimed at ultra-broadband flat spectral broadening (supercontinuum generation) by nonlinear propagation through a normally dispersive optical fiber [43].

\section{Nonlinear Optical Time Division Demultiplexing at 640 Gbit/s Using Flat-Top Pulse Gating}

3.1. Flat-Top Pulse Gating Schemes Based on a Single-LPG Shaper. The single-LPG scheme for flat-top optical pulse shaping described in Section 2.1 above has been successfully employed to generate optimized gating (control) pulses for error-free and jitter-tolerant nonlinear switching in optical time division multiplexing (OTDM) systems operating up to $640 \mathrm{Gbit} / \mathrm{s}$ [24-26]. The use of flat-top control pulses for demultiplexing in OTDM schemes has proved greatly advantageous as compared with the use of Gaussian-like pulses: flat-top pulse gating offers significant improvements in receiver sensitivity and timing-jitter tolerance. We reiterate that increasing tolerance to timing jitter in the system is of critical importance for serial data transmission in telecommunication links operating at rates of $160 \mathrm{Gbit} / \mathrm{s}$ and above.

In a first experimental demonstration [25], picosecond flat-top pulses generated from a single-LPG detuned differ- entiator were employed as gating pulses in a 640 -to- $10 \mathrm{Gbit} / \mathrm{s}$ demultiplexing configuration and they were demonstrated to provide an $\sim 13 \mathrm{~dB}$ improvement in receiver sensitivity as compared to the use of Gaussian-like control pulses and a jitter tolerance of $\sim 500 \mathrm{fs}$, nearly $30 \%$ of the one-bit time window.

Figure 9 shows a schematic of the experimental setup. It consists of three principal parts that will be described subsequently: the flat-top pulse generator, the transmitter, and the demultiplexer, a nonlinear optical loop mirror (NOLM).

The NOLM consists of a Sagnac interferometer [28] containing a $50 \mathrm{~m}$ long section of a highly nonlinear fiber (HNLF, dispersion slope $\sim 0.018 \mathrm{ps} / \mathrm{nm}^{2} \mathrm{~km}$, zero dispersion at $1554 \mathrm{~nm}$, and nonlinear coefficient of $\left.\gamma \sim 10.5 \mathrm{~W}^{-1} \mathrm{~km}^{-1}\right)$. The signal propagates inside the loop in both (opposite) directions and interferes at the NOLM's input/output coupler. Without the presence of the gating signal, the phase difference experienced by light propagating in both directions is zero, which results in constructive interference at the input port of the NOLM, that is, the signal is reflected back. In the presence of the gating pulse that propagates unidirectionally in the NOLM only, the copropagating signal light experiences a Kerr-effect-induced nonlinear phase shift, which introduces a phase difference between signals propagating in both directions. Adjusting the parameters (e.g., the gating signal intensity) to produce a phase difference equal to $\pi$, the signal, which is overlapped with the gating pulse, is directed to the NOLM output port. For optimum operation, the gating signal has to be propagated simultaneously with the target data pulse. To minimize dispersion-induced delays, 


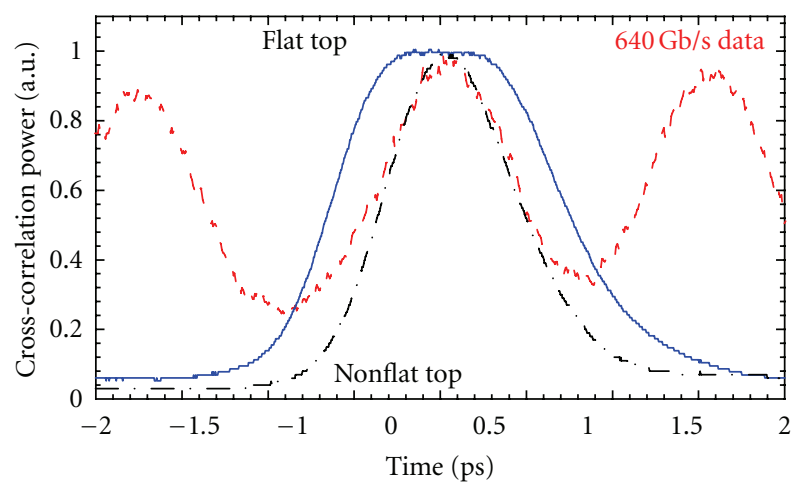

FIGURE 10: Cross-correlation of the data (dashed), flat-top (solid), and nonflat-top (dash-dot) gating pulses.

the signal and gating pulses should be respectively located at each different side of the zero-dispersion wavelength of the $\operatorname{NOLM}(1554 \mathrm{~nm})$. Nonetheless, any delay between these two pulses (caused, e.g., by the timing jitter) severely degrades the performance. As the tolerance to the timing jitter scales with the one-bit time slot duration, higher repetition rate systems are generally more timing-jitter sensitive.

The flat-top pulse generator consists of an ERGO (erbium glass oscillator) pulse source emitting 1.2 ps full-widthhalf-maximum (FWHM) pulses at $10 \mathrm{GHz}$ at $1543 \mathrm{~nm}$ with rms timing jitter of $90 \mathrm{fs}$ (measured by the von der Linde method [44], integrating the phase noise spectrum from $1 \mathrm{kHz}$ to $1 \mathrm{GHz}$ ). These pulses are further soliton compressed using an optical amplifier in saturation to obtain $450 \mathrm{fs}$ pulses (measured with an autocorrelator). Subsequently, the pulses are propagated through the LPG filter. The used LPG is $110 \mathrm{~mm}$ long with a period of $530 \mu \mathrm{m}$ and is made in a standard SMF-28 fiber using $\mathrm{CO}_{2}$ laser irradiation and the point-by-point technique [36]. Its coupling strength that is close to the full coupling condition (zero transmission at the resonance wavelength) is slightly tunable using axial strain [37]. The FWHM of the LPG notch is $6 \mathrm{~nm}$ in power transmission. Following optimization of the frequency detuning between the input pulse and LPG resonance, flat-top pulses that give a cross-correlation FWHM of $\sim 1.4$ ps are obtained at the LPG output. The sampling pulse used for the crosscorrelation is a $500 \mathrm{fs}$ FWHM Gaussian-shaped pulse (measured using an autocorrelator). The temporal characteristics of the flat-top pulses at the LPG shaper output are shown in Figure 10. The flat-top width of the pulses measured at $98 \%$ of the pulse cross-correlation peak power is measured to be $550 \mathrm{fs}$. Considering the relatively long duration of the sampling pulse used for the cross-correlation, the actual pulse flat-top part is expected to be slightly larger and the FWHM slightly smaller compared to the cross-correlation values. Due to an unwanted photoinduced birefringence of our in-house made LPG-based filter, a polarization controller is employed to operate the LPG-based filter within one principal axis of birefringence. The energetic efficiency of the filtering process (ratio of the signal powers at the LPG output and input) was measured to be $24 \%$.

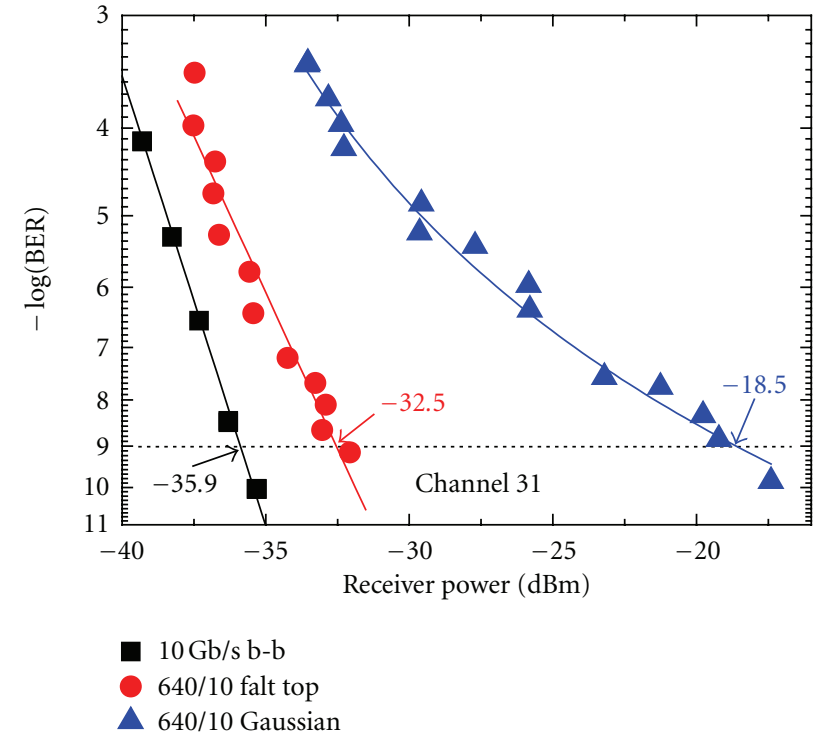

FIGURE 11: BER characteristics: back-to-back (squares), using flattop pulses (circles), and using nonflat-top pulses (triangles). The demultiplexing results are from the same data channel.

The transmitter consists of another $10 \mathrm{GHz}$ ERGO source emitting at $1557 \mathrm{~nm}$, a pulse compressor based on HNLF followed by SMF-28 standard fiber giving pulses of $530 \mathrm{fs}$ FWHM duration (estimated from autocorrelation measurements) with measured rms timing jitter of $70 \mathrm{fs}$. The pulse train is data modulated at $10 \mathrm{Gbit} / \mathrm{s}\left(2^{7}-1 \mathrm{PRBS}\right)$ and optical time division multiplexed (OTDM) up to $640 \mathrm{Gbit} / \mathrm{s}$, see cross-correlation trace of the data signal in Figure 10. Thus, the $640 \mathrm{Gbit} / \mathrm{s}$ serial data signal is composed by 64 channels, each at $10 \mathrm{Gbit} / \mathrm{s}$. Please note that in practice, the data modulated pulses are multiplexed to $40 \mathrm{Gbit} / \mathrm{s}$ before compression, in order to avoid unwanted dispersive broadening in the first multiplexer-stages. The multiplexer (MUX) is polarization and PRBS maintaining (for $2^{7}-1$ PRBS word length) with a $\left(2^{7}-1\right) / 2$ bits fiber delay in each MUX stage. The cross-correlation measurement of the multiplexed $640 \mathrm{Gbit} / \mathrm{s}$ data signal evidences that the individual data channels have been temporally aligned with the correct distance to neighboring channels, that is, about $1.57 \mathrm{ps}$, and the pulses are adequately narrow to obtain a reasonable resolution of the individual channels using the $500 \mathrm{fs}$ reference pulses.

The amplified flat-top gating signal and properly delayed transmitter signal are launched into the NOLM demultiplexer. The $10 \mathrm{Gbit} / \mathrm{s}$ demultiplexed signal at the NOLM output is filtered by a band-pass filter $(1.4 \mathrm{~nm})$ to filter out noise and residual gating signal at $1543 \mathrm{~nm}$ before being analyzed using a bit error ratio (BER) analyzer. We used a freerunning ERGO as a master clock and then o/e converted its pulses to lock all other subsystems to it. This reduced the timing jitter to $70 \mathrm{fs}$ rms for the data and $90 \mathrm{fs}$ for the clock pulses. For the system evaluation (results shown in Figure 11), we measure the back-to-back sensitivity at $10 \mathrm{Gbit} / \mathrm{s}$ first. Subsequently, we characterize the $640-\mathrm{Gbit} / \mathrm{s}$ setup: we measure power penalty using the optical gate based 


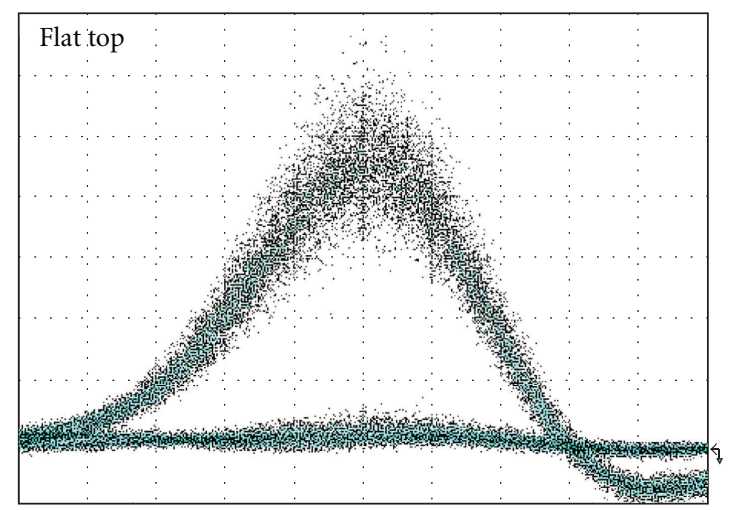

(a)

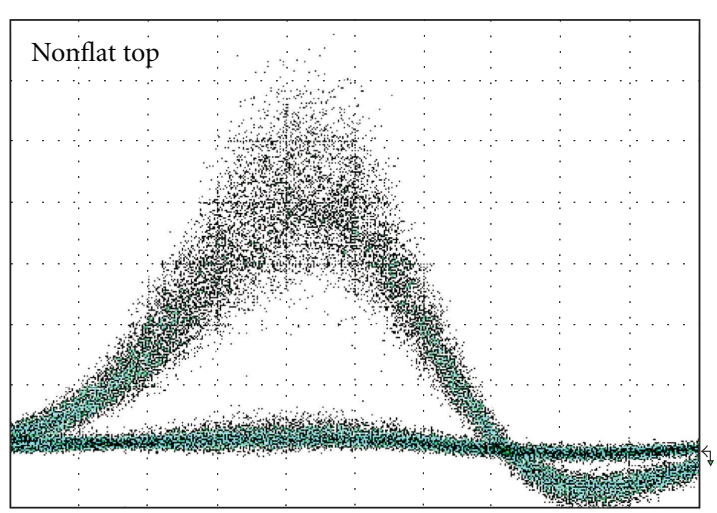

(b)

FIGURE 12: Eye diagrams obtained for flat-top and non-flat-top gating pulses.

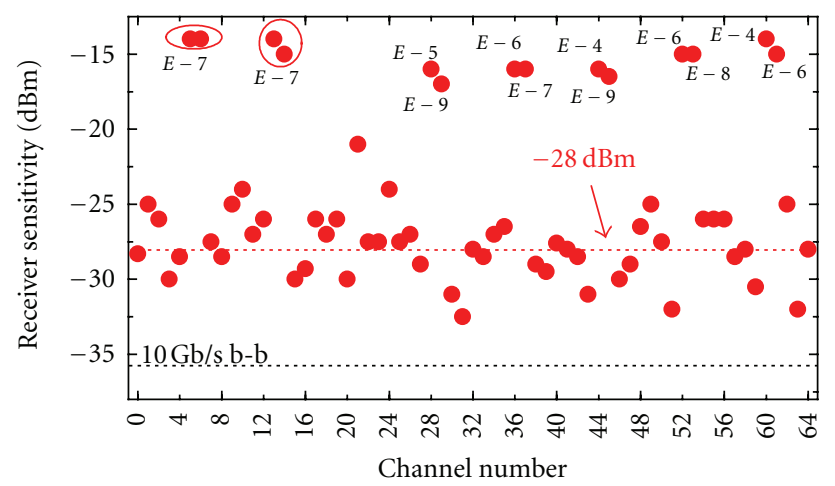

FIGURE 13: Demultiplexing of all 64 tributary channels using flattop gating pulses.

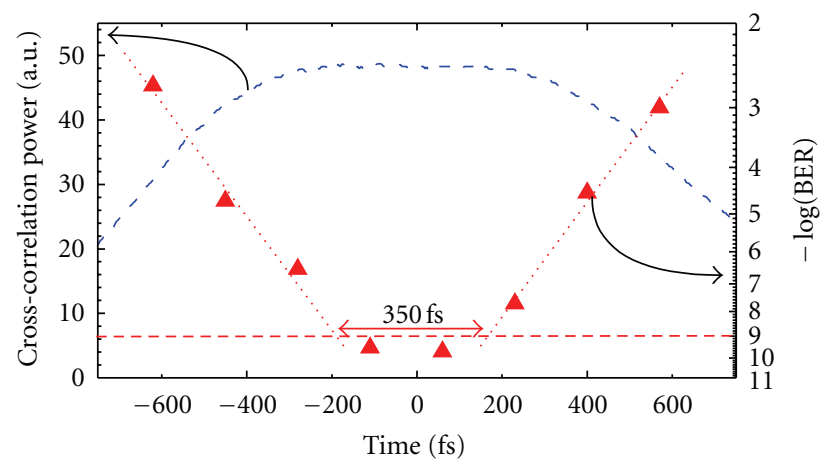

FIGURE 14: BER timing-jitter tolerance (triangles) and intensity profile of the used flat-top pulse (dashed).

on the flat-top pulses and for comparison with a non-flattop pulse. The non-flat-top reference pulse is prepared by simply detuning the LPG, which enables us to remain on the same data channel, yielding a fair comparison of the two pulse types. The non-flat-top reference pulse is a Gaussianlike shaped 900 fs FWHM pulse, see plot in Figure 10.

From Figure 11 we see that the use of non-flat-top gating pulses results in an additional power penalty of $13 \mathrm{~dB}$ for the error-free level of BER $=10^{-9}$ compared to the flat-top case, where the penalty is only $3.5 \mathrm{~dB}$ with respect to the $10 \mathrm{Gbit} / \mathrm{s}$ back to back. Moreover, there is an error floor using nonflat-top pulses indicating that the system is limited by relative timing jitter, which is over $110 \mathrm{fs}$ rms in our system (considering independent transmitter and gating signals timing jitter of $70 \mathrm{fs}$ and $90 \mathrm{fs}$, resp.). This is confirmed by the fact that the use of the flat-top gating pulses with a flat-top width fivetimes wider than the system rms timing jitter does not lead to the appearance of an error floor. That is, the non-flat-top error floor does not stem from interference from neighboring channels but only from timing mismatch with the data pulse caused by their relative jitter. For this reason flat-top pulses perform better, because their shape combined with the nonlinear mechanism of performing the demultiplexing process leads to a quasisquare switching window. This is further confirmed by the eye diagrams that are shown in Figure 12. We clearly see that using the non-flat-top gating pulses leads to amplitude noise, which is typical for timing jitter-limited systems. Again, influence of this phenomenon is considerably reduced when flat-top gating pulses are used. All these measurements were carried out on the same data channel.

Furthermore, we measure the system performance for all 64 tributary channels. Figure 13 shows the sensitivities for all 64 channels when using the flat-top pulses to gate with. We see that it is possible to resolve all channels and demultiplex them. But since some of the channels partially overlap, we see that some pairs of channels are not as good as the rest, and 12 of the 64 channels are not error-free due to the suboptimum MUX alignment. This is purely a feature of the multiplexer and is consequently not associated with the gating pulses. In fact the flat-top-pulses clearly reveal the quality of the multiplexed signal in a much better way than the non-flattop pulse would have been able to with its large penalty. Furthermore, with 52 channels error free, it can be concluded that the flat-top pulses are unambiguously appropriate for $640 \mathrm{Gbit} / \mathrm{s}$ operation.

The previous data clearly shows that the used flat-top gating pulses are capable of mitigating the system timing jitter being over $110 \mathrm{fs}$ rms. To find a maximum value of the timing jitter that can be tolerated by the demultiplexer with the available flat-top pulses, we increase the power at 


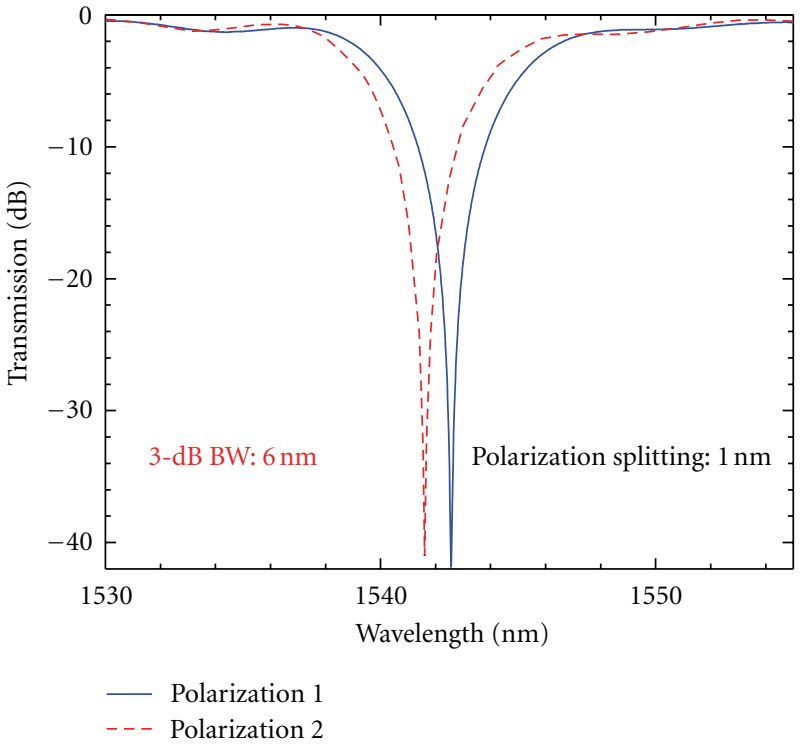

(a)

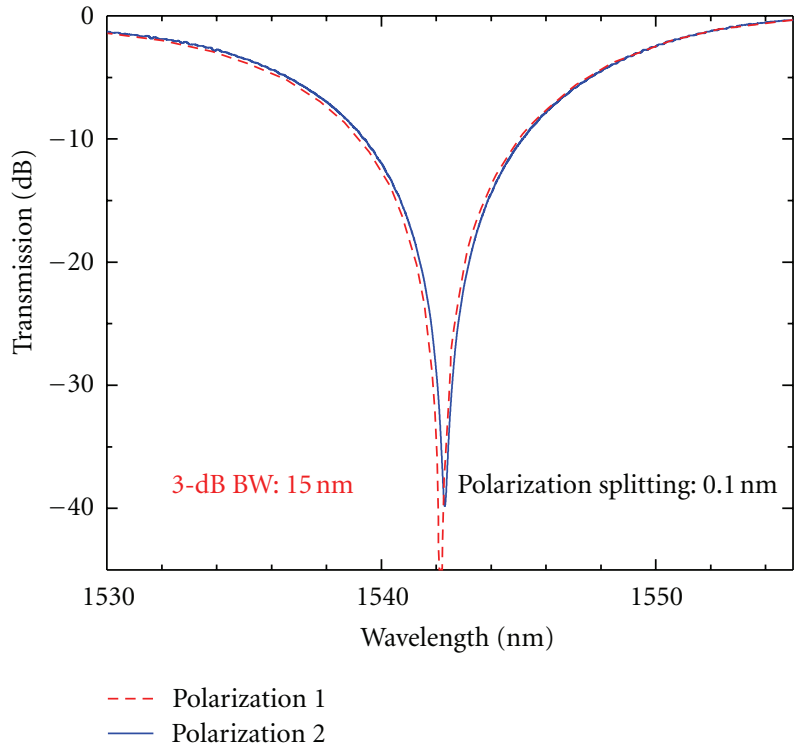

(b)

FIGURE 15: Measured spectral transmission responses of the LPG differentiators used (a) on the [25] (results shown in Figures 10-14) and (b) on the [26] (results shown in Figure 16) for the two orthogonal input light polarization states.

the receiver by $5 \mathrm{~dB}$ over the error-free level and vary the delay between the data and gating pulses. Subsequently, we measure the BER for different levels of this delay, Figure 14. The flat-top waveform is also shown in order to visualize the relation between the gating pulse flat-top duration and the amount of delay that can be tolerated for error-free operation (with BER $<10^{-9}$ ). We see that an additional time displacement of $350 \mathrm{fs}$ can be tolerated. Together with the system jitter of $110 \mathrm{fs}$, this gives almost $500 \mathrm{fs}$ tolerance or $30 \%$ of the $640 \mathrm{Gbit} / \mathrm{s}$ time slot, which is a value very close to the flat part of the flat-top pulse of $550 \mathrm{fs}$. For non-flat-top gating pulses, the system does not tolerate any additional timing jitter, indicating that the system is already limited by its timing jitter of $110 \mathrm{fs}$ rms.

In addition to the above critical advantages, it has been also demonstrated that the use of flat-top control pulses in a nonlinear time-domain demultiplexing system may help in achieving polarization-independent operation [45]. The latter is a highly desired feature in an optical demultiplexer, enabling it to process incoming optical data signals with arbitrary states of polarization, as it is usually the case in practical systems. For this purpose, the flat-top pulse-shaping element should be polarization independent. As mentioned above, LPG-based pulse shapers with significantly reduced polarization dependence can be created by fabricating the LPG devices through a two-mirror assisted $\mathrm{CO}_{2}$ laser radiation configuration, which yields azimuthally symmetric illumination of the fiber [41], greatly reducing the photo-induced birefringence in the fabricated LPGs. Figure 15(b) shows the measured transmission spectral response for the two orthogonal light polarization states of a single LPG differentiator fabricated using this improved configuration [26]. As anticipated, a nearly polarization-independent re- sponse is achieved (polarization splitting $\sim 0.1 \mathrm{~nm}$ ), in sharp contrast to the conventional single-side illuminated LPG differentiator (polarization splitting $\sim 1 \mathrm{~nm}$ ), see Figure 15(a) showing the measured spectral responses for the two orthogonal light polarization states of the LPG used in the nonlinear switching experiments described above [25]. Notice that the newly fabricated LPG differentiator also exhibits a significantly larger operation bandwidth (FWHM bandwidth of the resonance notch $\sim 15 \mathrm{~nm}$ ) than that used in our previous experiments $(\sim 6 \mathrm{~nm})$. This enables the generation of shorter flat-top pulse waveforms ( 840-fs FWHM from a $500 \mathrm{fs}$ input Gaussian-like pulse) with an improved intensityprofile quality, leading to a significantly improved BER performance when employed for 640-to-10 Gbit/s demultiplexing in the nonlinear switch [26]. In particular, the optimized LPG differentiator yields less than $0.5 \mathrm{~dB}$ receiver sensitivity penalty relative to the $10 \mathrm{Gbit} / \mathrm{s}$ back-to-back measurements, see results in Figure 16, as compared with the $>3 \mathrm{~dB}$ penalty measured for the previous LPG filters, see results in Figure 11 above. A total timing jitter tolerance of $\sim 310 \mathrm{fs}$ has been experimentally estimated when using the newly generated flat-top pulse waveform [26]. The observed decrease in the jitter tolerance (as compared with the results obtained with the previous flat-top pulse, $\sim 500 \mathrm{fs}$ ) can be attributed to the fact that the new pulse has a shorter flat-top section.

3.2. Flat-Top Pulse Gating Schemes Based on Double LPG for Polarization-Independent Demultiplexing. Polarizationindependent (PI) operation is one of the most desired features of an optical demultiplexer, enabling it to process incoming optical data signals with arbitrary states of polarizations. But nonlinear effects such as cross-phase modulation (XPM) or four wave mixing (FWM), which 

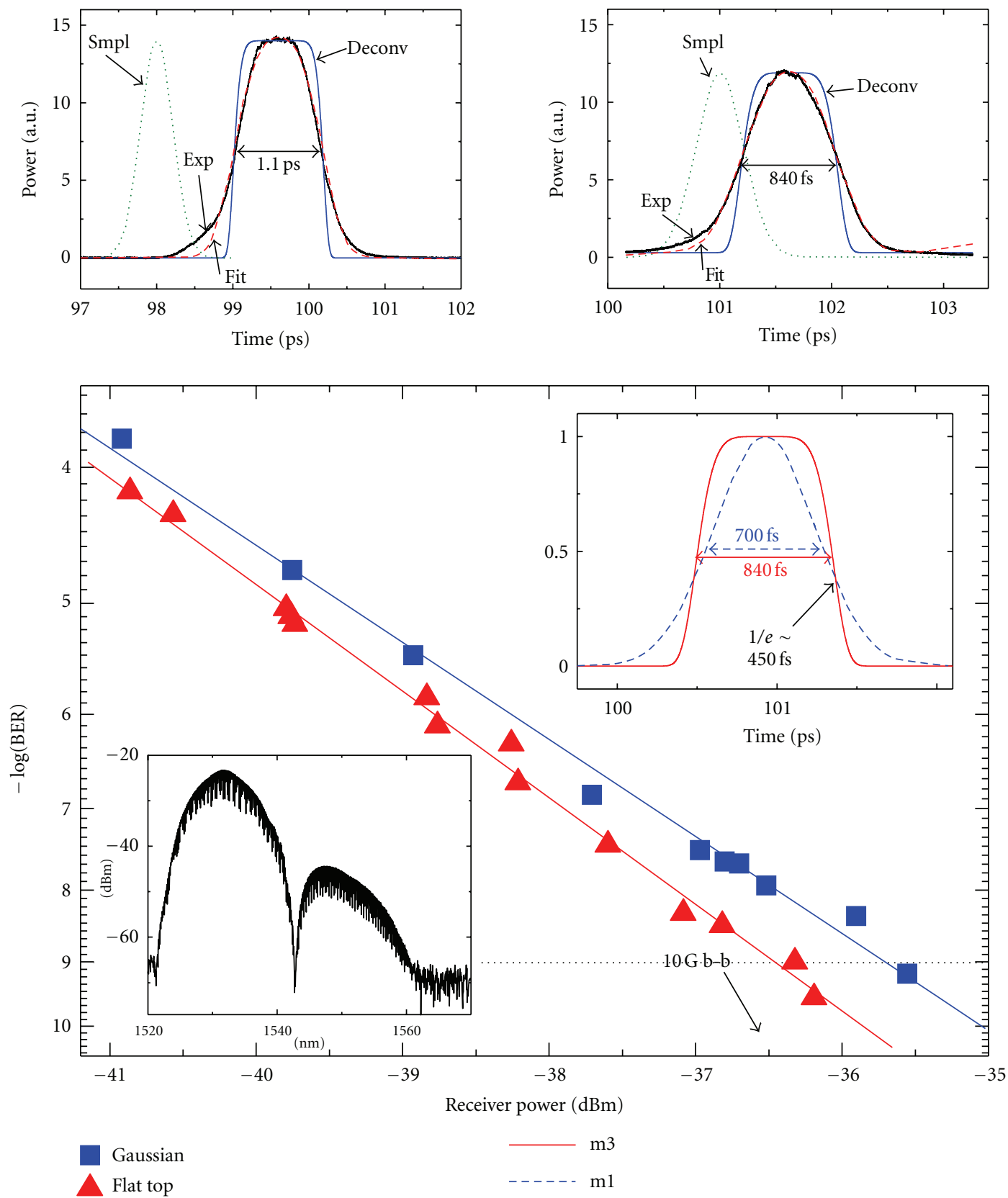

FIGURE 16: Flat-top pulses and $640 \mathrm{Gbit} / \mathrm{s}$ demultiplexing with subpicosecond flat-top pulse. Top: measured cross-correlation traces of the generated flat-top pulses of various widths and deconvoluted waveforms. Bottom: BER performance with and without flat-top pulse. Insets show the spectral (lower left) profile of the flat-top pulse and the temporal (upper right) corresponding waveforms of the flat-top superGaussian (or order $M=3$ ) and Gaussian $(m=1)$ control pulses.

are used in ultrahigh-speed all-optical demultiplexing, are inherently polarization dependent. This is the case also for the standard NOLM, since it is based on an XPM-induced phase shift.

The principle for canceling the data polarization-dependence of the NOLM is based on the careful use of its periodic power transfer function without introduction of any structural modifications [45]. The operation is shown in Figure 17. The polarization states are shown to the left: the control polarization is set to a linear state along the $y$-axis, and the data is in a random (elliptical) state with fieldcomponents $E_{x}$ and $E_{y}$ along the $x$ - and $y$-axes, respectively. The XPM phase shift $\Delta \varphi_{y}$ induced by the control pulse on the parallel $E_{y}$-component is three-times larger compared to the XPM phase shift $\Delta \varphi_{x}$ induced on the perpendicular component $E_{x}$. The power transfer function of the NOLM as a function of the phase-shift is shown to the right. It is possible to find conditions under which the XPM phaseshifts of the two data-polarization components $E_{y}$ and $E_{x}$, $\Delta \varphi_{y}$ and $\Delta \varphi_{x}=1 / 3 \Delta \varphi_{y}$, respectively, give rise to equal 


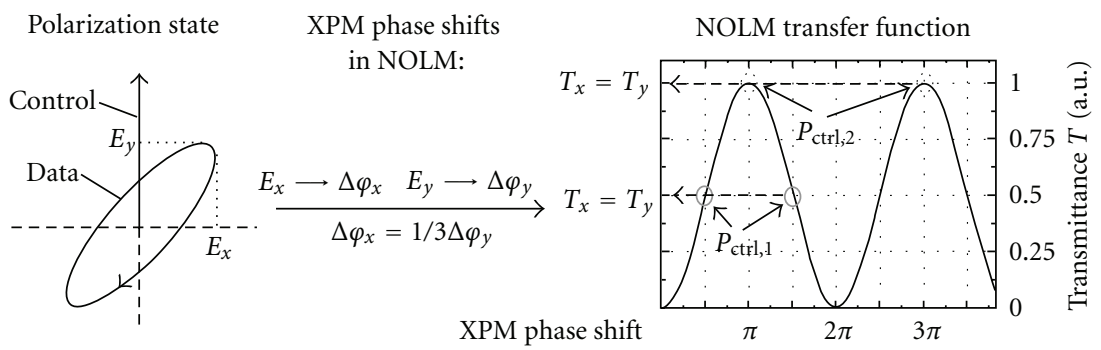

FIGURE 17: Principle behind polarization-independent operation of a standard NOLM.

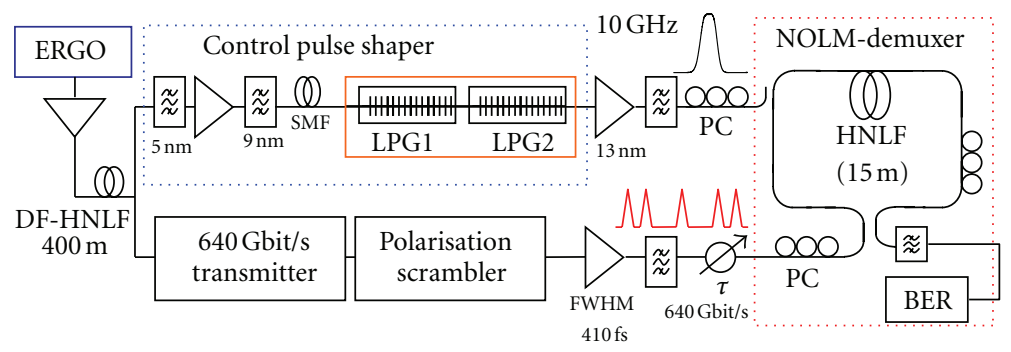

FIGURE 18: Setup for PI-NOLM operation.

transmissions $T_{y}$ and $T_{x}$. This will result in a polarization-independent switching operation and it occurs at $\left(\Delta \varphi_{x} ; \Delta \varphi_{y}\right)=$ $(\pi / 2 ; 3 \pi / 2)$ and $\left(\Delta \varphi_{x} ; \Delta \varphi_{y}\right)=(\pi ; 3 \pi)$. These conditions can be achieved by carefully adjusting the average control pulse power $P_{\text {ctrl }}$, since the XPM-phase shift is proportional to $P_{\text {ctrl }}$. It is easy to understand that PI operation of the NOLM would benefit from the utilization of flat-top control pulses. This is related to the fact that a constant control pulse power would result in a constant phase shift along the data channel to be demultiplexed.

The double-LPG flat-top pulse shaper described in Section 2.3 above has been successfully employed for polarization-independent nonlinear switching in a 640-to$10 \mathrm{Gbit} / \mathrm{s}$ demultiplexing experiment [27]. Figure 18 shows the experimental setup for the PI-NOLM operation using the cascaded LPG pair as a flat-top pulse shaper (see detailed descriptions on this specific pulse shaper in Section 2.3 above). An ERGO pulse source runs at $10 \mathrm{GHz}$ and emits $1.5 \mathrm{ps}$ wide pulses at $1542 \mathrm{~nm}$, which are used to create a supercontinuum in $400 \mathrm{~m}$ of dispersion flattened highly nonlinear fiber (DF-HNLF). The output from the DF-HNLF is used to generate the control and data signal by using two optical band-pass filters centered respectively at 1536 and $1555 \mathrm{~nm}$.

The double-LPG filter is used for generation of a $1.2 \mathrm{ps}$ flat-top pulse centered at $1536 \mathrm{~nm}$ from an input $700 \mathrm{fs}$ Gaussian-like pulse. The generated flat-top pulse is subsequently used as control in an NOLM with $15 \mathrm{~m} \mathrm{HNLF}$ (dispersion slope $S \approx 0.015 \mathrm{ps} /\left(\mathrm{nm}^{2} \mathrm{~km}\right)$, zero dispersion at $\lambda_{0}=$ $1545 \mathrm{~nm}$, and nonlinear coefficient $\left.\gamma \approx 10.5 \mathrm{~W}^{-1} \mathrm{~km}^{-1}\right)$. The data is a $640 \mathrm{Gbit} / \mathrm{s}$ OTDM signal (PRBS $2^{7}-1$, single polarization) centered at $1555 \mathrm{~nm}$, with $410 \mathrm{fs}$ wide pulses. The data is subsequently demultiplexed down to $10 \mathrm{Gbit} / \mathrm{s}$ for BER characterization.

The polarization state of the data signal is randomized in a polarization scrambler running at $113 \mathrm{kHz}$. The standard
NOLM operation (nonpolarization insensitive operation) is reached for $P_{\text {ctrl }}=26.5 \mathrm{dBm}$. When the scrambler is turned on the demultiplexed eye is severely deteriorated (Figure 19(a)), independently on the polarization state of the control. When $P_{\text {ctrl }}$ is increased to $27.6 \mathrm{dBm}$ and the control polarization is optimized, then the NOLM works in PI mode. With the scrambler on, the eye remains open as seen in Figure 19(b). By using a slow photodetector it is possible to measure $0.7 \mathrm{~dB}$ residual polarization dependence of the PINOLM operation when the scrambler is on.

Figure 20 shows the BER measurement for the standard and PI-NOLM operation. As can be seen, when the scrambler is off, the power sensitivity (at BER $=10^{-9}$ ) is the same for both cases. When the scrambler is turned on, then the standard NOLM exhibits an error floor above BER $=10^{-7}$. In contrast, the PI-NOLM shows just a power penalty of $\sim 1 \mathrm{~dB}$. These measurements confirm both the flat-top pulse enhanced performance of the nonlinear switch and the PI operation of the NOLM.

\section{Conclusions}

In this paper, we have reviewed some recent work on the design and fabrication of all-fiber (LPG-based) picosecond and subpicosecond flat-top optical pulse shapers and their application for nonlinear optical telecommunication data processing, particularly demultiplexing of OTDM serial data using NOLM-based switches with flat-top gating pulses, at bit rates up to $640 \mathrm{Gbit} / \mathrm{s}$. The reviewed set of experiments demonstrate error-free 640 -to-10 Gbit/s data signal demultiplexing over the 64 OTDM channels with a significantly improved performance over the use of Gaussian-like control/gating pulses, for example, in terms of receiver sensitivity (up to $\sim 13 \mathrm{~dB}$ ), timing-jitter tolerance (up to $\sim 500 \mathrm{fs}$, corresponding to $\sim 30 \%$ of the OTDM signal bit period), and optimized polarization insensitivity. 


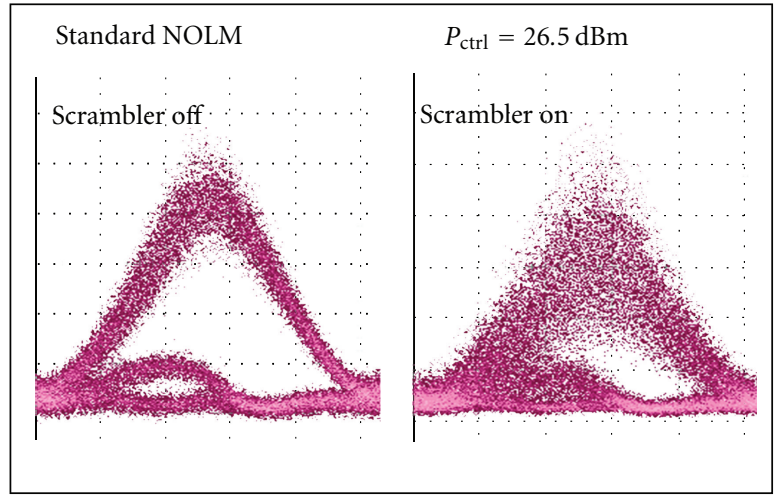

(a)

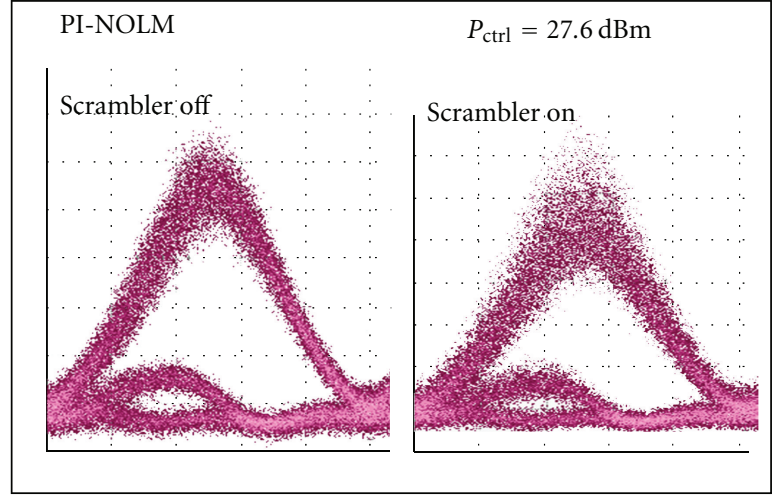

(b)

FIGURE 19: Eye diagrams for the demultiplexed $10 \mathrm{Gbit} / \mathrm{s}$ signal for the (a) standard, and (b) PI-NOLM operations, with polarization scrambler on and off.

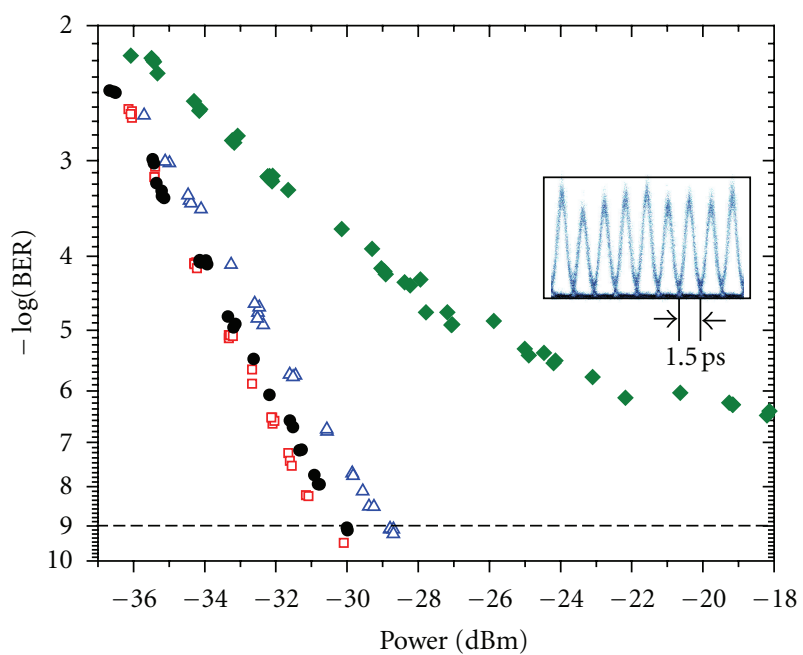

口 PI-NOLM, scrambler off

$\triangle$ PI-NOLM, scrambler on

- Standard NOLM, scrambler off

- Standard NOLM, scrambler on

Figure 20: BER curves for the standard and PI-NOLM operation. Inside: $640 \mathrm{Gbit} / \mathrm{s}$ eye diagram.

The described LPG-based optical pulse-shaping methods can be readily used for generation of even shorter flat-top pulse waveforms, opening the path for the creation of optical time-division demultiplexing platforms with an optimized performance at $1.2 \mathrm{Tbit} / \mathrm{s}$ and above. Moreover, other temporal pulse shapes of practical interest (e.g., triangular or parabolic waveforms) could be generated in the subpicosecond regime using similar LPG approaches, potentially enabling the implementation of other pulse shaping-enhanced telecommunication signal processing/generation operations (e.g., optimized wavelength conversion, signal copying, timeto-wavelength mapping, high-repetition rate femtosecond pulse generation, etc.) at unprecedented bit rates, that is, at $640 \mathrm{Gbit} / \mathrm{s}$ and above.

\section{Acknowledgments}

This paper was supported in part by the Natural Sciences and Engineering Research Council (NSERC) of Canada and by the Les Fonds Québécois de la Recherche sur la Nature et les Technologies (FQRNT). It was also supported by the European Research Council (ERC) starter grant project SOCRATES and the Danish National Research Council funded project NOSFERATU. OFS Fitel Denmark Aps is gratefully acknowledged for providing the nonlinear fibers used in these experiments.

\section{References}

[1] A. M. Weiner, "Femtosecond optical pulse shaping and processing," Progress in Quantum Electronics, vol. 19, no. 3, pp. 161-237, 1995.

[2] F. Parmigiani, P. Petropoulos, M. Ibsen, and D. J. Richardson, "All-optical pulse reshaping and retiming systems incorporating pulse shaping fiber Bragg grating," Journal of Lightwave Technology, vol. 19, pp. 746-752, 2001.

[3] J. H. Lee, P. C. Teh, P. Petropoulos, M. Ibsen, and D. J. Richardson, "All-optical modulation and demultiplexing systems with significant timing jitter tolerance through incorporation of pulse-shaping fiber Bragg gratings," IEEE Photonics Technology Letters, vol. 14, no. 2, pp. 203-205, 2002.

[4] J. H. Lee, L. K. Oxenløwe, M. Ibsen et al., "All-optical TDM data demultiplexing at $80 \mathrm{~Gb} / \mathrm{s}$ with significant timing Jitter tolerance using a fiber bragg grating based rectangular pulse switching technology," Journal of Lightwave Technology, vol. 21, no. 11, pp. 2518-2523, 2003.

[5] S. Watanabe, R. Ludwig, F. Futami et al., "Ultrafast all-optical 3R-regeneration," IEICE Transactions on Electronics, vol. E87C, no. 7, pp. 1114-1118, 2004.

[6] L. K. Oxenløwe, M. Galili, A. T. Clausen, and P. Jeppesen, "Generating a square switching window for timing jitter tolerant $160 \mathrm{~Gb} / \mathrm{s}$ demultiplexing by the optical Fourier transform technique," in Proceedings of the 32nd European Conference on Optical Communications (ECOC '06), Cannes, France, 2006, paper We2.3.4.

[7] T. Otani, T. Miyazaki, and S. Yamamoto, "Optical 3R regenerator using wavelength converters based on electroabsorption 
modulator for all-optical network applications," IEEE Photonics Technology Letters, vol. 12, no. 4, pp. 431-433, 2000.

[8] A. I. Latkin, S. Boscolo, R. S. Bhamber, and S. K. Turitsyn, "Optical frequency conversion, pulse compression and signal copying using triangular pulses," in Proceedings of the European Conference on Optical Communication (ECOC '08), Brussels, Belgium, 2008, paper Mo.3.F.4.

[9] F. Parmigiani, M. Ibsen, T. T. Ng, L. Provost, P. Petropoulos, and D. J. Richardson, "An efficient wavelength converter exploiting a grating-based saw-tooth pulse shaper," IEEE Photonics Technology Letters, vol. 20, no. 17, pp. 1461-1463, 2008.

[10] F. Parmigiani, C. Finot, K. Mukasa et al., "Ultra-flat SPMbroadened spectra in a highly nonlinear fiber using parabolic pulses formed in a fiber Bragg grating," Optics Express, vol. 14, no. 17 , pp. 7617-7622, 2006.

[11] T. Kurokawa, H. Tsuda, K. Okamoto et al., "Time-spaceconversion optical signal processing using arrayed-waveguide grating," Electronics Letters, vol. 33, no. 22, pp. 1890-1891, 1997.

[12] P. Petropoulos, M. Ibsen, A. D. Ellis, and D. J. Richardson, "Rectangular pulse generation based on pulse reshaping using a superstructured fiber Bragg grating," Journal of Lightwave Technology, vol. 19, no. 5, pp. 746-752, 2001.

[13] Y. Park, M. H. Asghari, T. J. Ahn, and J. Azaña, “Transformlimited picosecond pulse shaping based on temporal coherence synthesization," Optics Express, vol. 15, no. 15, pp. 9584 9599, 2007.

[14] Y. Park, M. Kulishov, R. Slavík, and J. Azaña, "Picosecond and sub-picosecond flat-top pulse generation using uniform long-period fiber gratings," Optics Express, vol. 14, no. 26, pp. 12671-12678, 2006.

[15] R. Slavík, Y. Park, and J. Azaña, “Tunable dispersion-tolerant picosecond flat-top waveform generation using an optical differentiator," Optics Express, vol. 15, no. 11, pp. 6717-6726, 2007.

[16] R. Kashyap, Fiber Bragg Gratings, Academic Press, San Diego, Calif, USA, 2nd edition, 2009.

[17] M. Kulishov and J. Azaña, "Ultrashort pulse propagation in uniform and nonuniform waveguide long-period gratings," Journal of the Optical Society of America A, vol. 22, no. 7, pp. 1319-1333, 2005.

[18] R. Slavík, M. Kulishov, Y. Park, and J. Azaña, "Long-periodfiber-grating-based filter configuration enabling arbitrary linear filtering characteristics," Optics Letters, vol. 34, no. 7, pp. 1045-1047, 2009.

[19] M. Kulishov and J. Azaña, "Long-period fiber gratings as ultrafast optical differentiators," Optics Letters, vol. 30, no. 20, pp. 2700-2702, 2005.

[20] R. Slavík, Y. Park, M. Kulishov, R. Morandotti, and J. Azaña, "Ultrafast all-optical differentiators," Optics Express, vol. 14, no. 22, pp. 10699-10707, 2006.

[21] R. Slavík, Y. Park, D. Krčmařík, and J. Azaña, "Stable all-fiber photonic temporal differentiator using a long-period fiber grating interferometer," Optics Communications, vol. 282, no. 12, pp. 2339-2342, 2009.

[22] R. Slavík, Y. Park, and J. Azaña, "Long-period fiber-gratingbased filter for generation of picosecond and subpicosecond transform-limited flat-top pulses," IEEE Photonics Technology Letters, vol. 20, no. 10, pp. 806-808, 2008.

[23] E. Palushani, H. Hu, L. K. Oxenløwe et al., " 640 Gb/s timing tolerant demultiplexing using a cascaded long-period fiber grating pulse shaper," in Proceedings of the 35th European Conference on Optical Communication (ECOC '09), Vienna, Austria, 2009, paper Tu4.3.3.
[24] R. Slavík, L. K. OxenlØwe, M. Galili et al., "Demultiplexing of 320-Gb/s OTDM data using ultrashort flat-top pulses," IEEE Photonics Technology Letters, vol. 19, no. 22, pp. 1855-1857, 2007.

[25] L. K. Oxenløwe, R. Slavík, M. Galili et al., “640 Gb/s timing jitter-tolerant data processing using a long-period fiber-grating-based flat-top pulse shaper," IEEE Journal on Selected Topics in Quantum Electronics, vol. 14, no. 3, Article ID 4538055, pp. 566-572, 2008.

[26] L. K. Oxenløwe, M. Galili, H. C. M. Mulvad, R. Slavík, A. T. Clausen, and P. Jeppesen, "Polarisation-independent sub-picosecond flat-top pulse generation for ultra-fast 640 Gbit/s gating," in Proceedings of the Optical Fiber Conference (OFC '09), San Diego, Calif, USA, March 2009, paper OWS5.

[27] E. Palushani, H. Hu, M. Galili et al., " 640 Gbit/s polarisationindependent demultiplexing in a standard nonlinear-opticalloop-mirror using a cascaded long-period grating pulse shaper," in Proceedings of the 23rd Annual Photonics Society Meeting, Denver, Colo, USA, November 2010, paper TuM2.

[28] T. Yamamoto, E. Yoshida, and M. Nakazawa, "Ultrafast nonlinear optical loop mirror for demultiplexing 640Gbit/s TDM signals," Electronics Letters, vol. 34, no. 10, pp. 1013-1014, 1998.

[29] A. I. Siahlo, A. T. Clausen, L. K. Oxenløwe, J. Seoane, and P. Jeppesen, " $640 \mathrm{~Gb} / \mathrm{s}$ OTDM transmission and demultiplexing using a NOLM with commercially available highly non-linear fiber," in Proceedings of the Conference on Lasers and ElectroOptics (CLEO '05), vol. 2, pp. 883-885, 2005, paper CTuO1.

[30] H. G. Weber, C. Schubert, S. Ferber et al., "Single channel $1.28 \mathrm{Tbit} / \mathrm{s}$ and $2.56 \mathrm{Tbit} / \mathrm{s}$ DQPSK transmission," Electronics Letters, vol. 42, pp. 178-179, 2006.

[31] S. Watanabe, "Optical signal processing using nonlinear fibers," Journal of Optical and Fiber Communications Reports, vol. 3, no. 1, pp. 1-24, 2006.

[32] D. Zibar, L. K. Oxenløwe, H. C. H. Mulvad et al., "The impact of gating timing jitter on a $160 \mathrm{~Gb} / \mathrm{s}$ demultiplexer," in Proceedings of the Optical Fiber Conference (OFC '06), vol. 2006, 2006, paper OTuB2.

[33] E. Tangdiongga, Y. Liu, H. de Waardt et al., "All-optical demultiplexing of 640 to $40 \mathrm{Gbits} / \mathrm{s}$ using filtered chirp of a semiconductor optical amplifier," Optics Letters, vol. 32, no. 7, pp. 835-837, 2007.

[34] M. H. Asghari and J. Azaña, "Proposal and analysis of a reconfigurable pulse shaping technique based on multi-arm optical differentiators," Optics Communications, vol. 281, no. 18, pp. 4581-4588, 2008.

[35] A. M. Vengsarkar, P. J. Lemaire, J. B. Judkins, V. Bhatia, T. Erdogan, and J. E. Sipe, "Long-period fiber gratings as bandrejection filters," Journal of Lightwave Technology, vol. 14, no. 1, pp. 58-65, 1996.

[36] D. D. Davis, T. K. Gaylord, E. N. Glytsis, S. G. Kosinski, S. C. Mettler, and A. M. Vengsarkar, "Long-period fibre grating fabrication with focused $\mathrm{CO}_{2}$ laser pulses," Electronics Letters, vol. 34, no. 3, pp. 302-303, 1998.

[37] R. Slavík, "Extremely deep long-period fiber grating made with $\mathrm{CO}_{2}$ laser," IEEE Photonics Technology Letters, vol. 18, no. 16, pp. 1705-1707, 2006.

[38] T. Erdogan and V. Mizrahi, "Characterization of UV-induced birefringence in photosensitive Ge-doped silica optical fibers," Journal of the Optical Society of America B, vol. 11, no. 10, pp. 2100-2105, 1994.

[39] L. Lepetit, G. Chériaux, and M. Joffre, "Linear technique of phase measurement by femtosecond spectral interferometry for applications in spectroscopy," Journal of the Optical Society of America B, vol. 12, no. 12, pp. 2467-2474, 1995. 
[40] Y. Park, F. Li, and J. Azaña, "Characterization and optimization of optical pulse differentiation using spectral interferometry," IEEE Photonics Technology Letters, vol. 18, no. 17, pp. 1798$1800,2006$.

[41] V. Grubsky and J. Feinberg, "Fabrication of axially symmetric long-period gratings with a carbon dioxide laser," IEEE Photonics Technology Letters, vol. 18, no. 21, pp. 2296-2298, 2006.

[42] Y. Park, J. Azaña, and R. Slavík, "Ultrafast all-optical first- and higher-order differentiators based on interferometers," Optics Letters, vol. 32, no. 6, pp. 710-712, 2007.

[43] D. Krčmařík, R. Slavík, Y. G. Park, and J. Azaña, "Nonlinear pulse compression of picosecond parabolic-like pulses synthesized with a long period fiber grating filter," Optics Express, vol. 17, no. 9, pp. 7074-7087, 2009.

[44] D. von der Linde, "Characterization of the noise in continuously operating mode-locked lasers," Applied Physics B, vol. 39, no. 4, pp. 201-217, 1986.

[45] H. C. H. Mulvad, M. Galili, L. K. Oxenløwe, A. T. Clausen, L. Grüner-Nielsen, and P. Jeppesen, "Polarization-independent high-speed switching in a standard non-linear optical loop mirror," in Proceedings of the Optical Fiber Conference (OFC '08), San Diego, Calif, USA, 2008, paper OMN3. 

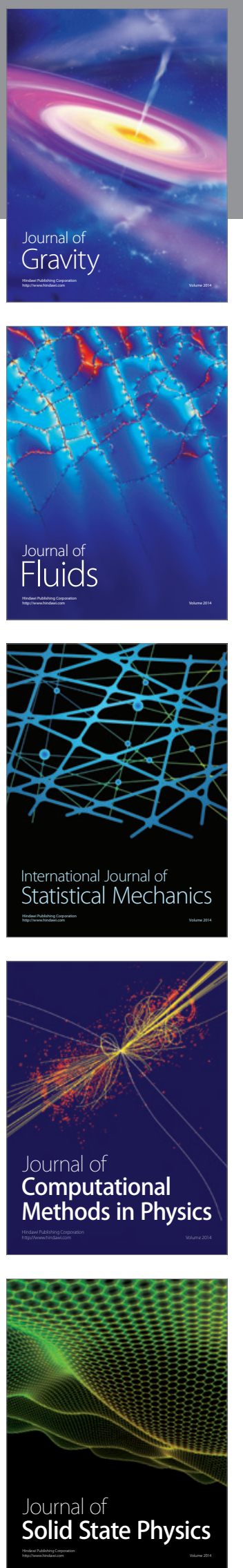

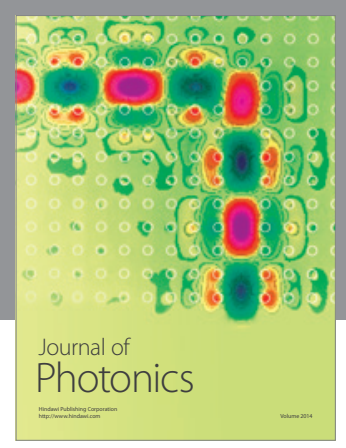

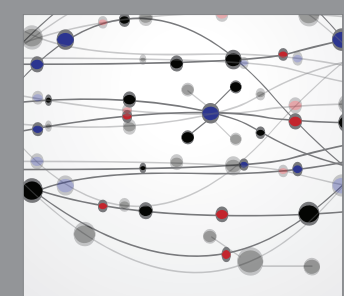

The Scientific World Journal
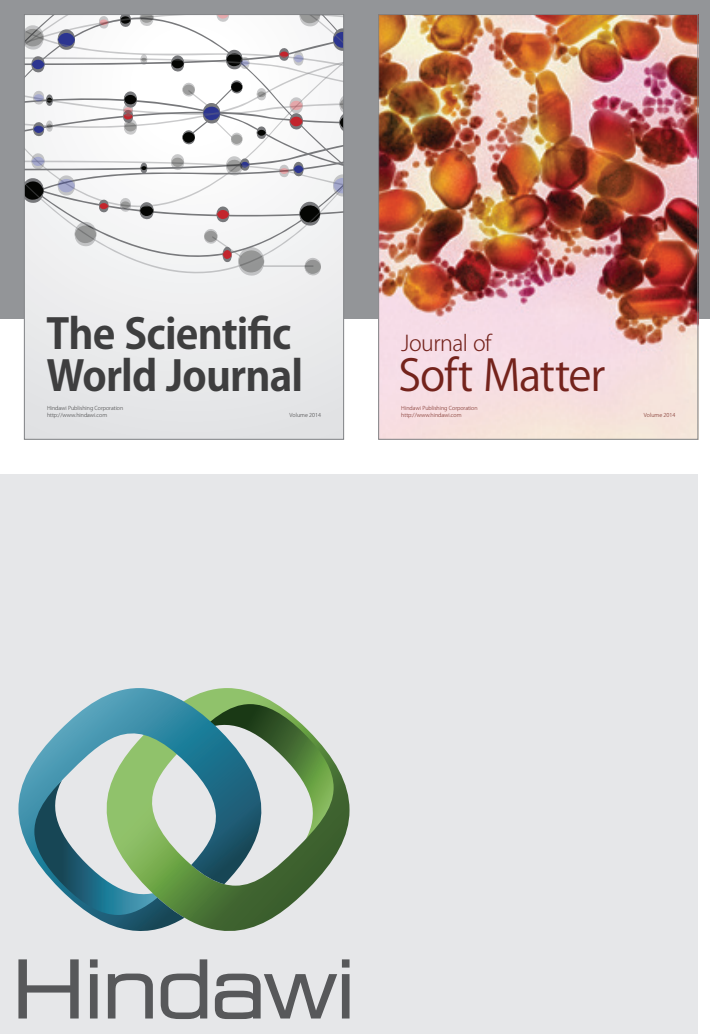

Submit your manuscripts at

http://www.hindawi.com
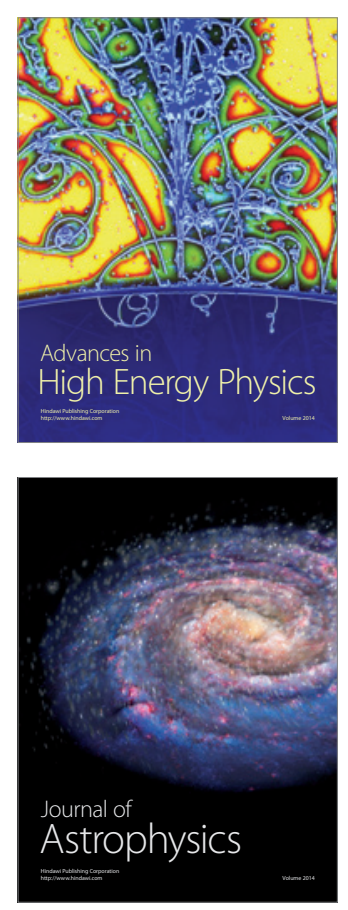
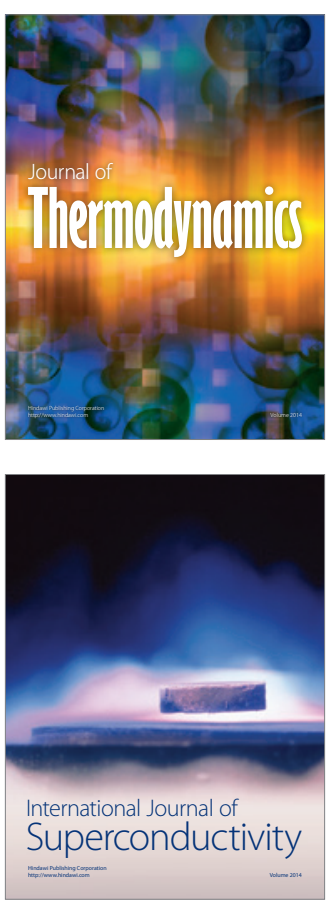
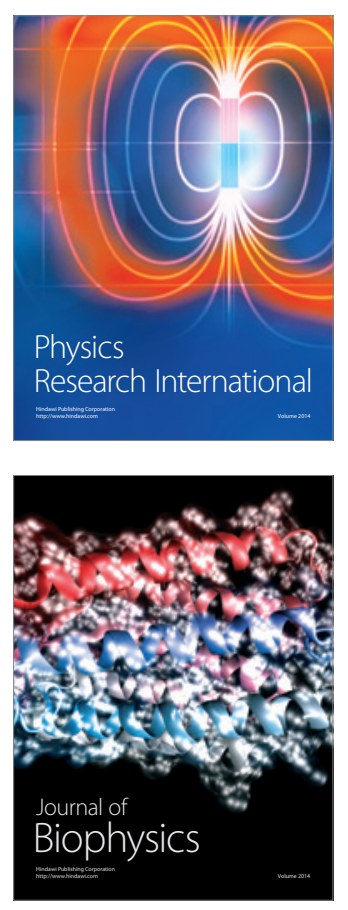
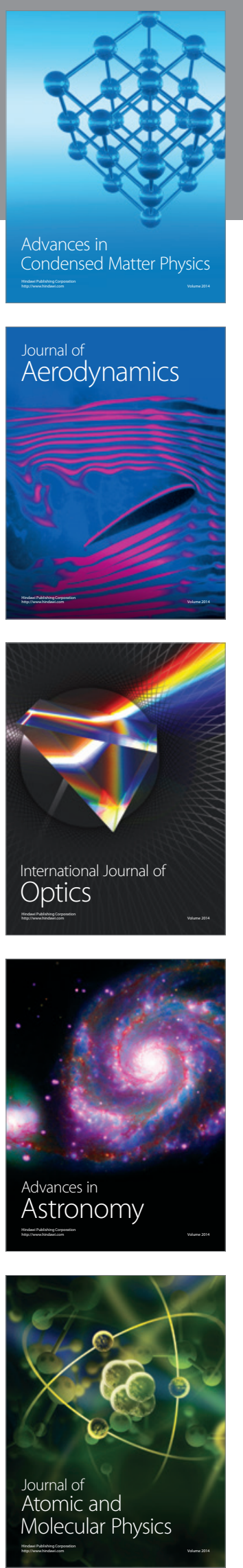